University of Nebraska - Lincoln

DigitalCommons@University of Nebraska - Lincoln

Faculty Publications, Department of Physics and Astronomy

Research Papers in Physics and Astronomy

2020

\title{
Magnonic analog of the Edelstein effect in antiferromagnetic insulators
}

Bo Li

University of Nebraska - Lincoln, boliwalker@huskers.unl.edu

Alexander Mook

University of Basel, Switzerland, alexander.mook@unibas.ch

Aldo Raeliarijaona

University of Nebraska - Lincoln, araeliarijaona2@unl.edu

Alexey Kovalev

University of Nebraska - Lincoln, alexey.kovalev@unl.edu

Follow this and additional works at: https://digitalcommons.unl.edu/physicsfacpub

Part of the Condensed Matter Physics Commons

Li, Bo; Mook, Alexander; Raeliarijaona, Aldo; and Kovalev, Alexey, "Magnonic analog of the Edelstein effect in antiferromagnetic insulators" (2020). Faculty Publications, Department of Physics and Astronomy. 308. https://digitalcommons.unl.edu/physicsfacpub/308

This Article is brought to you for free and open access by the Research Papers in Physics and Astronomy at DigitalCommons@University of Nebraska - Lincoln. It has been accepted for inclusion in Faculty Publications, Department of Physics and Astronomy by an authorized administrator of DigitalCommons@University of Nebraska - Lincoln. 


\title{
Magnonic analog of the Edelstein effect in antiferromagnetic insulators
}

\author{
Bo Li $\odot,{ }^{1}$ Alexander Mook, ${ }^{2,3}$ Aldo Raeliarijaona, ${ }^{1}$ and Alexey A. Kovalev ${ }^{1}$ \\ ${ }^{1}$ Department of Physics and Astronomy and Nebraska Center for Materials and Nanoscience, University of Nebraska, \\ Lincoln, Nebraska 68588, USA \\ ${ }^{2}$ Institut für Physik, Martin-Luther-Universität Halle-Wittenberg, D-06099 Halle (Saale), Germany \\ ${ }^{3}$ Department of Physics, University of Basel, Klingelbergstrasse 82, CH-4056 Basel, Switzerland
}

(Received 3 October 2019; revised manuscript received 11 December 2019; published 31 January 2020)

\begin{abstract}
We investigate the nonequilibrium spin polarization due to a temperature gradient in antiferromagnetic insulators, which is the magnonic analog of the inverse spin-galvanic effect of electrons. We derive a linear-response theory of a temperature-gradient-induced spin polarization for collinear and noncollinear antiferromagnets, which comprises both extrinsic and intrinsic contributions. We apply our theory to several noncentrosymmetric antiferromagnetic insulators, i.e., to a one-dimensional antiferromagnetic spin chain, a single layer of kagome noncollinear antiferromagnet, e.g., $\mathrm{KFe}_{3}(\mathrm{OH})_{6}\left(\mathrm{SO}_{4}\right)_{2}$, and a noncollinear breathing pyrochlore antiferromagnet, e.g., $\mathrm{LiGaCr}_{4} \mathrm{O}_{8}$. The shapes of our numerically evaluated response tensors agree with those implied by the magnetic symmetry. Assuming a realistic temperature gradient of $10 \mathrm{~K} / \mathrm{mm}$, we find two-dimensional spin densities of up to $\sim 10^{6} \hbar / \mathrm{cm}^{2}$ and three-dimensional bulk spin densities of up to $\sim 10^{14} \hbar / \mathrm{cm}^{3}$, encouraging an experimental detection.
\end{abstract}

DOI: 10.1103/PhysRevB.101.024427

\section{INTRODUCTION}

Generation of nonequilibrium spin imbalance is increasingly important for the current spintronics research [1], especially in the context of nonequilibrium torques [2]. In metallic and semiconductor materials, spin-orbit coupling (SOC) facilitates the interplay between the orbital and spin degrees of freedom, which allows feasible electric manipulation of spins, e.g., for technological applications. One consequence of such coupling is the inverse spin-galvanic effect [3-5] which attracted considerable interest in recent years [6-17]. The nonequilibrium spin polarization contains an extrinsic part dependent on the transport relaxation time and an intrinsic part independent of the relaxation time [2], and it can lead to spin-orbit torques. Both fieldlike and dampinglike spin-orbit torques can arise due to the nonequilibrium spin polarization at interfaces between magnetic and nonmagnetic materials [18-24].

In ferromagnetic and antiferromagnetic insulators, magnons - the quantum quasiparticles carrying energy and spin-can mediate various transport phenomena. The Dzyaloshinskii-Moriya interaction (DMI) [25,26] in such systems can lead to magnon spin-momentum locking [27], magnon-mediated magnetization torques [28-30], and magnonic thermal Hall [31-42] and spin Nernst effects [29,43-52]. In Ref. [46], two of us speculated about a possibility of magnon-mediated spin polarization in insulating antiferromagnets lacking inversion symmetry.

In this work, we study the magnonic analog of the Edelstein effect by considering antiferromagnetic insulators [53]. The spin nonconservation in such systems can be caused by noncollinear spin order or spin-orbit interactions (e.g., resulting in Dzyaloshinskii-Moriya interactions or anisotropic exchange). We consider a linear response to the temperature gradient replicated by a pseudo-gravitational potential [54] in the magnon Hamiltonian. The final result for the magnonic spin polarization is separated into the extrinsic and intrinsic contributions [53]. We apply our theory to several models and discuss relevant material candidates. In one dimension, an antiferromagnetic spin chain with anisotropic nearest exchange and Rashba-like DMI serves as a toy model exhibiting both intrinsic and extrinsic contributions to the magnonic analog of the Edelstein effect. In two and three dimensions, we concentrate on realistic noncollinear antiferromagnets on the kagome and breathing pyrochlore lattices. From the magnetic point group, we establish the response tensor shapes which agree with our numerical results.

The paper is organized as follows. In Sec. II, we discuss the Holstein-Primakoff transformation of magnons in noncollinear antiferromagnets, introduce the spin-density operator for magnons, and discuss the diagonalization procedure. In Sec. III, we derive the expression for the magnonic spin polarization as a linear response to the temperature gradient. In Sec. IV, we discuss the symmetry constraints on the response tensor. In Sec. V, we apply our theory to an antiferromagnetic spin chain and to noncollinear antiferromagnets on the kagome and breathing pyrochlore lattices. We also estimate the nonequilibrium spin density using real material parameters. In Sec. VI, we perform atomistic spin dynamics simulations and compare with our results from the previous section. Finally, we conclude our discussion in Sec. VII with a summary and an outlook. Appendixes contain more detailed information about our derivations.

\section{HAMILTONIAN AND EIGENSTATES}

We consider a general Hamiltonian of the form

$$
H=\sum_{i, j}\left[J_{i j}^{\alpha \beta} S_{i}^{\alpha} S_{j}^{\beta}+\mathbf{D}_{i j} \cdot\left(\mathbf{S}_{i} \times \mathbf{S}_{j}\right)\right]+\sum_{i} H_{i},
$$


where $i, j$ label different lattice sites and $\alpha, \beta$ stand for different spin vector components, i.e., $x, y, z$. Moreover, $J_{i j}^{\alpha \beta}$ is the symmetric exchange energy between $\alpha, \beta$ spin components on two sites $i$ and $j$, while antisymmetric exchange is described by the DMI vector $\mathbf{D}_{i j}$ between spins on sites $i$ and $j$. Effects of the on-site anisotropy and magnetic field may also be included in our analysis via the last term, $H_{i}=$ $K_{i}\left(\mathbf{S}_{i} \cdot \hat{\mathbf{z}}_{i}\right)^{2}+\left(\mathbf{S}_{i} \cdot \mathbf{B}\right)$.

We proceed with the Holstein-Primakoff transformation [55] in the limit of large spin and map spin operators onto bosonic creation and annihilation operators, $a_{i}^{\dagger}$ and $a_{i}$ :

$$
\begin{aligned}
\mathbf{S}_{i}= & \sqrt{2 S-a_{i}^{\dagger} a_{i}} a_{i} \frac{\hat{\mathbf{x}}_{i}-i \hat{\mathbf{y}}_{i}}{2}+a_{i}^{\dagger} \sqrt{2 S-a_{i}^{\dagger} a_{i}} \frac{\hat{\mathbf{x}}_{i}+i \hat{\mathbf{y}}_{i}}{2} \\
& +\left(S-a_{i}^{\dagger} a_{i}\right) \hat{\mathbf{z}}_{i} .
\end{aligned}
$$

Here, the unit vectors $\hat{\mathbf{x}}_{i}, \hat{\mathbf{y}}_{i}$, and $\hat{\mathbf{z}}_{i}$ form a local coordinate frame at position $i$ with $\hat{\mathbf{z}}_{i}$ pointing along the ground-state spin direction of site $i$ determined by a particular magnetic ordering. Keeping the leading-order terms of bosonic operators, we obtain the bilinear Hamiltonian written in magnon particle-hole space as

$$
H=\frac{1}{2} \int d \mathbf{r} \Psi^{\dagger}(\mathbf{r}) \mathcal{H} \Psi(\mathbf{r}),
$$

where $\Psi(\mathbf{r})=\left(a_{1}(\mathbf{r}), \ldots, a_{N}(\mathbf{r}), a_{1}^{\dagger}(\mathbf{r}), \ldots, a_{N}^{\dagger}(\mathbf{r})\right)^{T}, \mathcal{H}$ is the real-space single-particle Hamiltonian describing noninteracting magnons, and $\mathbf{r}$ describes the coordinate of a magnetic unit cell containing $N$ atoms.

In momentum space, Hamiltonian (3) reads

$$
H=\frac{1}{2} \sum_{\mathbf{k}} \Psi_{\mathbf{k}}^{\dagger} \mathcal{H}_{\mathbf{k}} \Psi_{\mathbf{k}}
$$

where $\Psi_{\mathbf{k}}=\left(a_{1, \mathbf{k}}, \ldots, a_{N, \mathbf{k}}, a_{1,-\mathbf{k}}^{\dagger}, \ldots, a_{N,-\mathbf{k}}^{\dagger}\right)^{T}$. From the standard bosonic commutation relation $\left[a_{i, \mathbf{k}}, a_{j, \mathbf{k}}^{\dagger}\right]=\delta_{i j}$, it can be shown that

$$
\left[\Psi_{i, \mathbf{k}}, \Psi_{j, \mathbf{k}}^{\dagger}\right]=\left(\sigma_{3}\right)_{i j},
$$

where here and henceforth $\sigma_{i}(i=0,1,2,3)$ stands for the Pauli matrices acting in particle-hole space (with $\sigma_{0}$ being the identity matrix). Hamiltonian (4) can be diagonalized by a matrix $T_{\mathbf{k}}$, which introduces Bogoliubov quasiparticles: $\Gamma_{\mathbf{k}}=T_{\mathbf{k}}^{-1} \Psi_{\mathbf{k}}$ with $\Gamma_{\mathbf{k}}=\left(\gamma_{1, \mathbf{k}}, \ldots, \gamma_{N, \mathbf{k}}, \gamma_{1,-\mathbf{k}}^{\dagger}, \ldots, \gamma_{N,-\mathbf{k}}^{\dagger}\right)^{T}$. In terms of the eigenbasis, Eq. (4) reads

$$
H=\frac{1}{2} \sum_{\mathbf{k}} \Gamma_{\mathbf{k}}^{\dagger} \mathcal{E}_{\mathbf{k}} \Gamma_{\mathbf{k}}=\sum_{\mathbf{k}} \sum_{n=1}^{N} \varepsilon_{n, \mathbf{k}}\left(\gamma_{n, \mathbf{k}}^{\dagger} \gamma_{n, \mathbf{k}}+\frac{1}{2}\right),
$$

where $\mathcal{E}_{\mathbf{k}}=\operatorname{Diag}\left(\varepsilon_{1, \mathbf{k}}, \ldots, \varepsilon_{N, \mathbf{k}}, \varepsilon_{1,-\mathbf{k}}, \ldots, \varepsilon_{N,-\mathbf{k}}\right)$ is the eigenenergy matrix containing the eigenvalues $\varepsilon_{n, \mathbf{k}}$. By plugging $\Psi_{\mathbf{k}}=T_{\mathbf{k}} \Gamma_{\mathbf{k}}$ into Eq. (5) and recalling that the normal-mode quasiparticles are bosons as well, i.e., $\left[\Gamma_{i, \mathbf{k}}, \Gamma_{j, \mathbf{k}}^{\dagger}\right]=\left(\sigma_{3}\right)_{i j}$, we conclude that $T_{\mathbf{k}}$ is paraunitary:

$$
T_{\mathbf{k}}^{\dagger} \sigma_{3} T_{\mathbf{k}}=T_{\mathbf{k}} \sigma_{3} T_{\mathbf{k}}^{\dagger}=\sigma_{3} .
$$

To appreciate the differences to a unitary transformation, let us write the diagonalization in a more suggestive matrix form,

$$
T_{\mathbf{k}}^{\dagger} \mathcal{H}_{\mathbf{k}} T_{\mathbf{k}}=T_{\mathbf{k}}^{\dagger} \sigma_{3}\left(\sigma_{3} \mathcal{H}_{\mathbf{k}}\right) T_{\mathbf{k}}=\mathcal{E}_{\mathbf{k}}
$$

where $\sigma_{3} \mathcal{H}_{\mathbf{k}}$ can be regarded as a pseudo-Hermitian Hamiltonian. Although it violates hermiticity, it can still be diagonalized by different left and right eigenstates with corresponding real eigenvalues. $T_{\mathbf{k}}^{\dagger} \sigma_{3}$ and $T_{\mathbf{k}}$ are alignments of left and right eigenstates, respectively: the $l$ th entry of the $n$th left (right) eigenstate reads $\left\langle\left. u_{n, \mathbf{k}}^{L}\right|_{l}=\left(T_{\mathbf{k}}^{\dagger} \sigma_{3}\right)_{n l}\left[\left|u_{n, \mathbf{k}}^{R}\right\rangle_{l}=\left(T_{\mathbf{k}}\right)_{l n}\right]\right.$. Paraunitarity is then expressed as $\left\langle u_{m, \mathbf{k}}^{L} \mid u_{n, \mathbf{k}}^{R}\right\rangle=\left(\sigma_{3}\right)_{m n}$ and Eq. (8) is equivalent to $\left\langle u_{m, \mathbf{k}}^{L}\left|\sigma_{3} \mathcal{H}_{\mathbf{k}}\right| u_{n, \mathbf{k}}^{R}\right\rangle=\left(\mathcal{E}_{\mathbf{k}}\right)_{n n} \delta_{m n}$. Thus, the pseudo-Hermitian Hamiltonian matrix in terms of its eigenbasis reads $\sigma_{3} \mathcal{H}_{\mathbf{k}}=\sum_{n}\left(\mathcal{E}_{\mathbf{k}}\right)_{n n}\left|u_{n, \mathbf{k}}^{R}\right\rangle\left\langle u_{n, \mathbf{k}}^{L}\right|$, from which follows a pair of eigenequations [56],

$$
\begin{aligned}
\sigma_{3} \mathcal{H}_{\mathbf{k}}\left|u_{n, \mathbf{k}}^{R}\right\rangle & =\bar{\varepsilon}_{n, \mathbf{k}}\left|u_{n, \mathbf{k}}^{R}\right\rangle, \\
\left\langle u_{n, \mathbf{k}}^{L}\right| \sigma_{3} \mathcal{H}_{\mathbf{k}} & =\left\langle u_{n, \mathbf{k}}^{L}\right| \bar{\varepsilon}_{n, \mathbf{k}},
\end{aligned}
$$

where $\bar{\varepsilon}_{n, \mathbf{k}}=\left(\sigma_{3} \mathcal{E}_{\mathbf{k}}\right)_{n n}$. From here on, we will only refer to the right eigenstates as $\left|u_{n, \mathbf{k}}^{R}\right\rangle=\left|u_{n, \mathbf{k}}\right\rangle$, and their left partners can be always obtained from $\left\langle u_{n, \mathbf{k}}^{L}\right|=\left\langle u_{n, \mathbf{k}}\right| \sigma_{3}$.

Finally, note that the magnon basis possesses particlehole symmetry (PHS) $\Psi_{\mathbf{k}}^{\dagger}=\left(\sigma_{1} \Psi_{-\mathbf{k}}\right)^{T}$ so that the Hamiltonian obeys $\sigma_{1} H_{\mathbf{k}} \sigma_{1}=H_{-\mathbf{k}}^{*}$, which leads to $\bar{\varepsilon}_{n+N, \mathbf{k}}=-\bar{\varepsilon}_{n,-\mathbf{k}}$ and $\left|u_{n, \mathbf{k}}\right\rangle=e^{i \phi_{n}} \sigma_{1}\left|u_{n+N,-\mathbf{k}}\right\rangle^{*}$, where $\phi_{n}$ is a redundant phase factor.

Since we are interested in a nonequilibrium spin-density response to an external force, we must denote the spin-density operator in terms of the aforementioned magnonic variables. We introduce the $\mu=x, y, z$ component of the magnonic spindensity operator as [57]

$$
\Sigma_{\mu}=\frac{1}{V} \sum_{\mathbf{k}} \Psi_{\mathbf{k}}^{\dagger} \hat{\Sigma}_{\mu} \Psi_{\mathbf{k}}
$$

where

$$
\hat{\Sigma}_{\mu}=-\frac{1}{2} \operatorname{Diag}\left(z_{1}^{\mu}, \ldots, z_{N}^{\mu}, z_{1}^{\mu}, \ldots, z_{N}^{\mu}\right),
$$

and the unit vectors $z_{i}^{\mu}$ corresponding to directions of magnetic moments have been introduced in Eq. (2). We note that PHS implies $\left\langle u_{n, \mathbf{k}}\left|\hat{\Sigma}_{\mu}\right| u_{n, \mathbf{k}}\right\rangle=\left\langle u_{n+N,-\mathbf{k}}\left|\hat{\Sigma}_{\mu}\right| u_{n+N,-\mathbf{k}}\right\rangle$.

\section{LINEAR-RESPONSE THEORY}

In this section, we perform linear-response calculations of the nonequilibrium spin density with respect to a temperature gradient $\nabla_{\nu} T$, i.e.,

$$
\left\langle\Sigma_{\mu}\right\rangle=\chi_{\mu \nu} \nabla_{\nu} T=\left(\chi_{\mu \nu}^{\mathrm{ex}}+\chi_{\mu \nu}^{\mathrm{in}}\right) \nabla_{\nu} T,
$$

where we separated the response tensor $\chi_{\mu \nu}$ into extrinsic, $\chi_{\mu \nu}^{\mathrm{ex}}$, and intrinsic, $\chi_{\mu \nu}^{\mathrm{in}}$, parts.

We introduce a perturbation corresponding to a pseudogravitational potential $\phi(\mathbf{r})$ :

$$
H^{\prime}=\frac{1}{4} \int d \mathbf{r} \Psi^{\dagger}(\mathbf{r})(\mathcal{H} \phi(\mathbf{r})+\phi(\mathbf{r}) \mathcal{H}) \Psi(\mathbf{r}),
$$

where $\phi(\mathbf{r})=-T(\mathbf{r}) / T$. Up to the linear order, the spatial gradients of this potential replicate the presence of the temperature gradient in the system. In addition, the pseudogravitational potential also amends the spin-density operator $[54,58]$. This can be seen by considering a response to magnetic field in the presence of perturbation (14). The total macroscopic 
spin-density operator becomes

$$
\Sigma_{\mu}=\frac{1}{V} \int d \mathbf{r} \Psi^{\dagger}(\mathbf{r})\left(\hat{\Sigma}_{\mu}+\frac{\phi}{2} \hat{\Sigma}_{\mu}+\hat{\Sigma}_{\mu} \frac{\phi}{2}\right) \Psi(\mathbf{r}) .
$$

Thus, the nonequilibrium spin density contains two parts:

$$
\left\langle\Sigma_{\mu}\right\rangle_{\text {tot }}=\left\langle\Sigma_{\mu}\right\rangle_{\text {neq }}+\left\langle\delta \Sigma_{\mu}\right\rangle_{\text {eq }}=\left(K_{\mu \nu}+D_{\mu \nu}\right) \nabla_{\nu} \phi,
$$

where the term proportional to $K_{\mu \nu}$ corresponds to the unperturbed spin-density operator and it can be calculated within the Kubo linear-response formalism. The dipole contribution $D_{\mu \nu}$ is evaluated with respect to the equilibrium state as it originates from the correction to the spin density in Eq. (15) containing the temperature gradient.

We first calculate $K_{\mu \nu}$ within the Kubo linear-response formalism $[29,43]$ in which the spin accumulation is given by

$$
\left\langle\Sigma_{\mu}\right\rangle_{\text {neq }}=\lim _{\omega \rightarrow 0} \frac{1}{i \omega}\left[\Pi_{\mu \nu}(\omega)-\Pi_{\mu \nu}(0)\right] \nabla_{\nu} \phi,
$$

where

$$
\Pi_{\mu \nu}\left(i \omega_{m}\right)=-\int_{0}^{1 / k_{B} T} d \tau e^{i \omega_{m} \tau}\left\langle T_{\tau} \Sigma_{\mu}(\tau) J_{v}^{q}(0)\right\rangle,
$$

and $\omega_{m}$ is the bosonic Matsubara frequency. The $v$ component of the macroscopic heat current, $J_{v}^{q}=\frac{1}{V} \int d \mathbf{r} j_{v}^{q}(\mathbf{r})$, is derived from the heat current density $\boldsymbol{j}^{q}=\frac{1}{4} \Psi^{\dagger}(\mathbf{r})\left(\mathcal{H} \sigma_{3} \mathbf{v}+\right.$ $\left.\mathbf{v} \sigma_{3} \mathcal{H}\right) \Psi(\mathbf{r})$, with velocity $\mathbf{v}=i[\mathcal{H}, \mathbf{r}]$. The heat current density can be inferred from the continuity equation, i.e., $\dot{\rho}_{E}+$ $\boldsymbol{\nabla} \cdot \boldsymbol{j}^{q}=0$, with $\rho_{E}$ being the energy density of the system. In Appendix A, we provide the detailed calculation of the response tensor $K_{\mu \nu}$ divided into intraband and interband parts: $K_{\mu \nu}=K_{\mu \nu}^{\text {intra }}+K_{\mu \nu}^{\text {inter }}$, whose explicit forms read

$$
\begin{gathered}
K_{\mu \nu}^{\text {intra }}=\frac{1}{V} \sum_{\mathbf{k}} \sum_{n=1}^{2 N} \frac{1}{\Gamma_{n}}\left(\mathcal{J}_{\nu, \mathbf{k}}\right)_{n n}\left(\Sigma_{\mu, \mathbf{k}}\right)_{n n} \partial_{\varepsilon} n_{\mathrm{B}}\left[\bar{\varepsilon}_{\mathbf{k}, n}\right], \\
K_{\mu \nu}^{\text {inter }}=\frac{4}{V} \sum_{\mathbf{k}} \sum_{m \neq n} \frac{\operatorname{Im}\left[\left(\sigma_{3} \Sigma_{\mu, \mathbf{k}}\right)_{n m}\left(\sigma_{3} \mathcal{J}_{\nu, \mathbf{k}}\right)_{m n}\right] n_{\mathrm{B}}\left[\bar{\varepsilon}_{\mathbf{k}, n}\right]}{\left(\bar{\varepsilon}_{\mathbf{k}, n}-\bar{\varepsilon}_{\mathbf{k}, m}\right)^{2}},
\end{gathered}
$$

where $n_{\mathrm{B}}(x)=1 /\left(e^{x / k_{B} T}-1\right)$ is the Bose-Einstein distribution function, and we used notations $\Sigma_{\mu, \mathbf{k}}=T_{\mathbf{k}}^{\dagger} \hat{\Sigma}_{\mu} T_{\mathbf{k}}, \mathcal{J}_{\mathbf{k}}=$ $T_{\mathbf{k}}^{\dagger} \mathbf{J}_{\mathbf{k}}^{q} T_{\mathbf{k}}$, and $\mathbf{J}_{\mathbf{k}}^{q}=\frac{1}{4}\left(\mathcal{H}_{\mathbf{k}} \sigma_{3} \mathbf{v}_{\mathbf{k}}+\mathbf{v}_{\mathbf{k}} \sigma_{3} \mathcal{H}_{\mathbf{k}}\right)$ with $\mathbf{v}_{\mathbf{k}}=\frac{\partial \mathcal{H}_{\mathbf{k}}}{\partial \mathbf{k}}$. As can be seen from Eq. (19), the phenomenological spectrum broadening, given by $\Gamma_{n}$, is crucial for the intraband component, whereas it does not enter the intrinsic contribution. Plugging $\mathcal{J}_{\nu, \mathbf{k}}=\frac{1}{4}\left(\mathcal{E}_{\mathbf{k}} \sigma_{3} \tilde{v}_{\nu, \mathbf{k}}+\tilde{v}_{\nu, \mathbf{k}} \sigma_{3} \mathcal{E}_{\mathbf{k}}\right)$ with $\tilde{v}_{v, \mathbf{k}}=T_{\mathbf{k}}^{\dagger} v_{\nu} T_{\mathbf{k}}$ into Eq. (19) (see details in Appendix A), we obtain the intraband (extrinsic) response tensor:

$$
\chi_{\mu \nu}^{\mathrm{ex}}=\frac{1}{V T} \sum_{\mathbf{k}, n=1}^{N} \frac{1}{\Gamma_{n}}\left(\Sigma_{\mu, \mathbf{k}}\right)_{n n} v_{n \mathbf{k}, \nu} \varepsilon_{n, \mathbf{k}}\left[-\frac{\partial n_{\mathrm{B}}\left(\varepsilon_{n, \mathbf{k}}\right)}{\partial \varepsilon}\right],
$$

where $v_{n \mathbf{k}, v}=\left(\tilde{v}_{v, \mathbf{k}}\right)_{n n}=\frac{\partial \varepsilon_{n, \mathbf{k}}}{\partial k_{v}}$. This result can be also obtained from the Boltzmann transport theory with the relaxation time $\tau_{n}=1 /\left(2 \Gamma_{n}\right)$. The interband contribution in Eq. (20) can be reorganized as

$$
K_{\mu \nu}^{\mathrm{inter}}=\frac{1}{V} \sum_{\mathbf{k}, n=1}^{2 N}\left[-\left(\Omega_{n, \mathbf{k}}^{\Sigma}\right)_{\mu \nu} \bar{\varepsilon}_{n, \mathbf{k}}-\left(m_{n, \mathbf{k}}^{\Sigma}\right)_{\mu \nu}\right] n_{\mathrm{B}}\left(\bar{\varepsilon}_{n, \mathbf{k}}\right)
$$

where

$$
\begin{aligned}
\left(\Omega_{n, \mathbf{k}}^{\Sigma}\right)_{\mu \nu} & =\sum_{m(\neq n)} \frac{2 \operatorname{Im}\left[\left(\sigma_{3} \Sigma_{\mu, \mathbf{k}}\right)_{n m}\left(\sigma_{3} \tilde{v}_{v, \mathbf{k}}\right)_{m n}\right]}{\left(\bar{\varepsilon}_{n, \mathbf{k}}-\bar{\varepsilon}_{m, \mathbf{k}}\right)^{2}} \\
\left(m_{n, \mathbf{k}}^{\Sigma}\right)_{\mu \nu} & =\sum_{m(\neq n)} \frac{-\operatorname{Im}\left[\left(\sigma_{3} \Sigma_{\mu, \mathbf{k}}\right)_{n m}\left(\sigma_{3} \tilde{v}_{v, \mathbf{k}}\right)_{m n}\right]}{\left(\bar{\varepsilon}_{n, \mathbf{k}}-\bar{\varepsilon}_{m, \mathbf{k}}\right)} .
\end{aligned}
$$

Here $\left(\Omega_{n, \mathbf{k}}^{\Sigma}\right)_{\mu \nu}$ satisfies a relation $\left(\Omega_{n, \mathbf{k}}^{\Sigma}\right)_{\mu \nu}=\left(\Omega_{n+N,-\mathbf{k}}^{\Sigma}\right)_{\mu \nu}$ and a sum rule $\sum_{n=1}^{2 N}\left(\Omega_{n, \mathbf{k}}^{\Sigma}\right)_{\mu \nu}=0$.

The expression for $K_{\mu \nu}^{\text {inter }}$ is not yet the final result for the intrinsic response. We now show that it can be conveniently combined with the dipole contribution

$$
D_{\mu \nu}=\left\langle\frac{1}{V} \int d \mathbf{r} \Psi^{\dagger}(\mathbf{r}) \hat{\Sigma}_{\mu} r_{\nu} \Psi(\mathbf{r})\right\rangle_{\mathrm{eq}},
$$

where we used that $\left[\hat{\Sigma}_{v}, r_{v}\right]=0$. To calculate this term, we explicitly introduce a perturbation corresponding to an external magnetic field $\mathbf{B}(\mathbf{r})$ into Hamiltonian $H$ [58-60]:

$$
\hat{\mathcal{H}}_{B}=-[\mathbf{B}(\mathbf{r}) \cdot \hat{\boldsymbol{\Sigma}}+\hat{\boldsymbol{\Sigma}} \cdot \mathbf{B}(\mathbf{r})],
$$

where $\mathbf{B}(\mathbf{r})$ varies slowly in space, i.e., on a length scale much larger than the lattice constant. The dipole moment can then be found from a thermodynamic relation [58],

$$
D_{\mu \nu}=-\lim _{\mathbf{B} \rightarrow 0} \frac{\partial \Omega}{\partial\left(\partial_{r_{v}} B_{\mu}\right)},
$$

where $\Omega$ is the thermodynamic grand potential of the system and the limit of vanishing magnetic field has to be taken. Using the Maxwell relation

$$
\left(\frac{\partial D_{\mu \nu}}{\partial T}\right)_{\mathbf{B}, \partial_{\mathbf{r}} \mathbf{B}}=\left[\frac{\partial S}{\partial\left(\partial_{r_{v}} B_{\mu}\right)}\right]_{T, \mathbf{B}},
$$

we introduce an auxiliary quantity $\tilde{D}_{\mu \nu}=-\frac{\partial K}{\partial\left(\partial_{r_{\nu}} B_{\mu}\right)}$, where $K=\Omega+T S$ and

$$
\tilde{D}_{\mu \nu}=\frac{\partial\left(\beta D_{\mu \nu}\right)}{\partial \beta}
$$

From the auxiliary quantity $\tilde{D}_{\mu \nu}$ we can calculate $D_{\mu \nu}$. The former is calculated using the perturbation theory applied to

$$
K(\mathbf{r})=\frac{1}{2} \sum_{\mathbf{k}, n=1}^{2 N}\left(\sigma_{3}\right)_{n n} g\left(\bar{\varepsilon}_{n, \mathbf{k}}\right)\left\langle\psi_{n, \mathbf{k}}(\mathbf{r})|\hat{K}| \psi_{n, \mathbf{k}}(\mathbf{r})\right\rangle,
$$

where $\left|\psi_{n, \mathbf{k}}(\mathbf{r})\right\rangle=e^{i \mathbf{k} \cdot \mathbf{r}}\left|u_{n, \mathbf{k}}\right\rangle$. For a perturbation $\mathbf{B}(\mathbf{r})=$ $B / q \sin (\mathbf{q} \cdot \mathbf{r}) \hat{\mathbf{e}}_{\mu}$, with $\mathbf{q}=q \hat{\mathbf{e}}_{v}$, we obtain

$$
\tilde{D}_{\mu \nu}=\lim _{\mathbf{q} \rightarrow 0} \frac{-2}{V B} \int d \mathbf{x} \delta K(\mathbf{r}) \cos (\mathbf{q} \cdot \mathbf{r}),
$$

where only the leading-order correction $\delta K(\mathbf{r})$ due to the magnetic field is considered. It is obtained from the expansion:

$$
\begin{aligned}
\delta K(\mathbf{r})= & \frac{1}{2} \sum_{n \mathbf{k}} \delta g\left(\bar{\varepsilon}_{n \mathbf{k}}\right)\left(\sigma_{3}\right)_{n n}\left\langle\psi_{n \mathbf{k}}\left|\hat{K}_{0}\right| \psi_{n \mathbf{k}}\right\rangle \\
& -g\left(\bar{\varepsilon}_{n \mathbf{k}}\right)\left(\sigma_{3}\right)_{n n}\left\langle\psi_{n \mathbf{k}}\left|\hat{\mathcal{H}}_{B}\right| \psi_{n \mathbf{k}}\right\rangle \\
& +g\left(\bar{\varepsilon}_{n \mathbf{k}}\right)\left(\sigma_{3}\right)_{n n}\left(\left\langle\delta \psi_{n \mathbf{k}}\left|\hat{K}_{0}\right| \psi_{n \mathbf{k}}\right\rangle+\left\langle\psi_{n \mathbf{k}}\left|\hat{K}_{0}\right| \delta \psi_{n \mathbf{k}}\right\rangle\right),
\end{aligned}
$$


with

$$
\left|\delta \psi_{n \mathbf{k}}\right\rangle=\sum_{m \neq n} \frac{i B}{2 q}\left(\sigma_{3}\right)_{m m}\left[e^{i(\mathbf{k}+\mathbf{q}) \cdot \mathbf{r}}\left|u_{m, \mathbf{k}+\mathbf{q}}\right\rangle \frac{\left\langle u_{m, \mathbf{k}+\mathbf{q}}\left|\left(\Sigma_{\mu, \mathbf{k}}+\Sigma_{\mu, \mathbf{k}+\mathbf{q}}\right)\right| u_{n, \mathbf{k}}\right\rangle}{\bar{\varepsilon}_{n \mathbf{k}}-\bar{\varepsilon}_{m, \mathbf{k}+\mathbf{q}}}-(\mathbf{q} \rightarrow-\mathbf{q})\right],
$$

where $\Sigma_{\mu, \mathbf{k}}=e^{-i \mathbf{k} \cdot \mathbf{r}} \hat{\Sigma}_{\mu} e^{i \mathbf{k} \cdot \mathbf{r}}=\hat{\Sigma}_{\mu}$. After substituting Eq. (31) into Eq. (30) we find (details in Appendix A)

$$
\begin{aligned}
\tilde{D}_{\mu \nu}= & \frac{1}{V} \sum_{n \mathbf{k}} g\left(\bar{\varepsilon}_{n, \mathbf{k}}\right) \bar{\varepsilon}_{n \mathbf{k}}\left(\Omega_{n, \mathbf{k}}^{\Sigma}\right)_{\mu \nu}+\left[g\left(\bar{\varepsilon}_{n, \mathbf{k}}\right)+g^{\prime}\left(\bar{\varepsilon}_{n, \mathbf{k}}\right)\right. \\
& \left.\times \bar{\varepsilon}_{n, \mathbf{k}}\right]\left(m_{n, \mathbf{k}}^{\Sigma}\right)_{\mu \nu}
\end{aligned}
$$

where for quasiequilibrium magnons with nonzero chemical potential we should have $\bar{\varepsilon}_{n, \mathbf{k}} \rightarrow \bar{\varepsilon}_{n, \mathbf{k}}-\mu$. Utilizing this expression as well as Eq. (28), we obtain the dipole contribution:

$$
D_{\mu \nu}=\frac{1}{V} \sum_{n \mathbf{k}}\left[\left(\Omega_{n, \mathbf{k}}^{\Sigma}\right)_{\mu \nu} \int_{0}^{\bar{\varepsilon}_{n \mathbf{k}}} d \eta g(\eta)+\left(m_{n, \mathbf{k}}^{\Sigma}\right)_{\mu \nu} g\left(\bar{\varepsilon}_{n, \mathbf{k}}\right)\right]
$$

This result has to be combined with the Kubo part in Eq. (22) to give us the total intrinsic contribution:

$$
\chi_{\mu \nu}^{\text {in }}=\frac{2 k_{B}}{V} \sum_{n=1}^{N} \sum_{\mathbf{k}}\left(\Omega_{n, \mathbf{k}}^{\Sigma}\right)_{\mu \nu} c_{1}\left[n_{\mathrm{B}}\left(\varepsilon_{n, \mathbf{k}}\right)\right]
$$

where we used the notation $c_{1}(x)=(1+x) \ln (1+x)-$ $x \ln (x)$. Note that we have expressed Eq. (35) in particle space by utilizing the properties of $\left(\Omega_{n \mathbf{k}}^{\Sigma}\right)_{\mu \nu}$ [46]; see details in Appendix A.

Equations (21) and (35) are the main results of this section. In Sec. V, we use these formulas to make numerical predictions of the nonequilibrium spin density for several relevant models, including material candidates.

Before proceeding to subsequent discussions, some useful remarks about the response theory developed above are due. The validity of magnon representation is well established at low temperatures. Higher-order magnon-magnon interaction corrections to the theory start with $\mathcal{O}(1 / S)$ terms. These corrections include both effects from thermally activated processes and spontaneous decay $[61,62]$. The former are frozen in the low-temperature regime. The latter contribute to spectrum broadening and renormalization, both of which are further suppressed by the weak magnon-magnon coupling factor and restriction of energy and momentum conservation. The magnon-phonon scattering effects lead to the phenomenological broadening factor $\Gamma_{n}$ in our theory [63]. Higher-order corrections, such as vertex corrections, could in principle be important in some cases but their consideration goes beyond the scope of this paper. In a special case, when the magnonphonon coupling is strong enough, it may become necessary to explore the magnon-phonon hybrid system $[64,65]$ where our theory still applies by treating quasiparticles as a mixture of magnon and phonon.

\section{SYMMETRY CONSTRAINTS}

In this section, we discuss constraints on the magnon response tensor $\chi_{\mu \nu}$ posed by the symmetries. To generate the nonequilibrium spin density with magnons one needs a system in which spin is not conserved locally or globally, at least for one direction of the spin polarization. This is often the case in noncollinear antiferromagnets or in systems with Dzyaloshinskii-Moriya interactions. For example, for inversion symmetric systems spin density is globally conserved [46]. To see this, note that inversion symmetry implies $\mathcal{H}_{\mathbf{k}}=\mathcal{H}_{-\mathbf{k}}$, which leads to $T_{\mathbf{k}}=T_{-\mathbf{k}}, \mathcal{E}_{\mathbf{k}}=\mathcal{E}_{-\mathbf{k}}$ and $\mathbf{v}_{n, \mathbf{k}}=$ $-\mathbf{v}_{n,-\mathbf{k}}$. Substituting these relations into Eq. (21) results in $\chi_{\mu \nu}^{\mathrm{ex}}=-\chi_{\mu \nu}^{\mathrm{ex}}=0$. Furthermore, inversion symmetry also enforces the relation $\left(\Omega_{n, \mathbf{k}}^{\Sigma}\right)_{\mu \nu}=-\left(\Omega_{n,-\mathbf{k}}^{\Sigma}\right)_{\mu \nu}$, which results in $\chi_{\mu \nu}^{\text {in }}=-\chi_{\mu \nu}^{\text {in }}=0$, that is, in a vanishing intrinsic response. Below, in Sec. V, we show several examples of collinear and noncollinear systems in which spin can be generated, because inversion symmetry is broken.

In general, the response tensor will be constrained by symmetry operations of a specific material under consideration. The constraining relations can be readily found within the framework of linear-response theory $[66,67]$. Assuming that a system respects a symmetry operation represented by $g$, we find for an arbitrary operator $\hat{A}$ that $\left\langle g\left(\psi_{n \mathbf{k}}\right)|\hat{A}| g\left(\psi_{m \mathbf{k}}\right)\right\rangle=$ $\left\langle\psi_{n g(\mathbf{k})}\left|g^{-1} \hat{A} g\right| \psi_{m g(\mathbf{k})}\right\rangle$ when the operation is unitary, and $\left\langle g\left(\psi_{n \mathbf{k}}\right)|\hat{A}| g\left(\psi_{m \mathbf{k}}\right)\right\rangle=\left\langle\psi_{n g(\mathbf{k})}\left|g^{-1} \hat{A} g\right| \psi_{m g(\mathbf{k})}\right\rangle^{*}$, when the operation is antiunitary. Operators transform as $g^{-1} \hat{v}_{i} g=\sum_{j} R_{i j}^{v} \hat{v}_{j}$ and $g^{-1} \hat{\Sigma}_{i} g=\sum_{j} R_{i j}^{s} \hat{\Sigma}_{j}$, where $R^{v / s}$ is the corresponding matrix representation of $g$ with respect to the Cartesian components $\hat{v}_{j}$ or $\hat{\Sigma}_{j}$. We find $R^{v}= \pm R$ and $R^{s}= \pm \operatorname{det}(R) R$ where \pm refers to unitary $(+)$ or antiunitary $(-)$ symmetries, respectively. Under the above premises, the following symmetry requirements on elements of the response tensor arise:

$$
\begin{aligned}
& \chi_{\mu \nu}^{\mathrm{ex}}=\operatorname{det}(R) R_{\mu i} R_{v j} \chi_{i j}^{\mathrm{ex}}, \\
& \chi_{\mu \nu}^{\mathrm{in}}= \pm \operatorname{det}(R) R_{\mu i} R_{v j} \chi_{i j}^{\mathrm{in}},
\end{aligned}
$$

where \pm corresponds to unitary and antiunitary symmetry operations, respectively. Later on, we show that these two relations result in different shapes of the response tensors, which is useful for distinguishing extrinsic and intrinsic contributions. Notice that tensors $\chi_{\mu \nu}^{\mathrm{ex}}$ and $\chi_{\mu \nu}^{\mathrm{in}}$ transform differently under antiunitary operations which is a consequence of a complex factor in the expression for $\left(\Omega_{n, \mathbf{k}}^{\Sigma}\right)_{\mu \nu}$ corresponding to taking the imaginary part in Eq. (23). Given the transformation properties of velocity and spin, one finds that $\chi_{\mu \nu}^{\mathrm{ex}}$ is even and $\chi_{\mu \nu}^{\text {in }}$ is odd under the time-reversal transformation. Consequently, a reversal of the magnetic ordering causes $\chi_{\mu \nu}^{\text {in }}$ to flip sign while $\chi_{\mu \nu}^{\mathrm{ex}}$ is invariant under such transformation:

$$
\begin{aligned}
& \chi_{\mu \nu}^{\text {in }}\left[\left\{\mathbf{S}_{i}\right\}\right]=-\chi_{\mu \nu}^{\text {in }}\left[\left\{-\mathbf{S}_{i}\right\}\right], \\
& \chi_{\mu \nu}^{\mathrm{ex}}\left[\left\{\mathbf{S}_{i}\right\}\right]=\chi_{\mu \nu}^{\mathrm{ex}}\left[\left\{-\mathbf{S}_{i}\right\}\right] .
\end{aligned}
$$

Thus, it is possible to disentangle extrinsic from intrinsic contributions by measuring the nonequilibrium spin density for two antiferromagnetically ordered states related by the 
time-reversal transformation. Such approach has been used in studies of the spin Hall effect [68].

A short note on the similarity to the electronic Edelstein effect is in order. In principle, the latter also consists of a time-reversal even extrinsic and time-reversal odd intrinsic contribution $[67,69,70]$. However, since the Edelstein effect is, for historic reasons, mainly studied in nonmagnetic materials, the extrinsic contribution has been analyzed in more detail. On the other hand, since the very notion of a magnon is tied to magnetism, both contributions are, in general, expected to be of equal importance.

\section{MODELS}

In this section, we apply our theory to specific models. To obtain some intuition, we first focus on a toy model of collinear antiferromagnetic spin chain with anisotropic exchange and inversion asymmetry resulting in Rashba-type DMI. We then focus on more realistic noncollinear kagome and breathing pyrochlore antiferromagnets, for which we use material parameters established in the literature. To satisfy the requirement of inversion asymmetry, we assume that the kagome antiferromagnet can have interfacial inversion asymmetry, e.g., due to thin-film geometry in contact with another material. The breathing pyrochlore antiferromagnet has bulk inversion asymmetry. For details of the HolsteinPrimakoff transformations and explicit expressions of the magnon Hamiltonians, we refer the reader to Appendix B.

\section{A. Antiferromagnetic spin chain}

As a simple model, we first consider the antiferromagnetic spin chain shown in Fig. 1(a). Similar to Eq. (1), the Hamiltonian

$$
\begin{aligned}
H= & \sum_{i} \sum_{\nu= \pm 1}\left[J\left(\gamma S_{1, i}^{x} S_{2, i+\nu}^{x}+S_{1, i}^{y} S_{2, i+\nu}^{y}+\lambda S_{1, i}^{z} S_{2, i+\nu}^{z}\right)\right. \\
& \left.+D_{12}^{v} \mathbf{e}_{z} \cdot\left(\mathbf{S}_{1, i} \times \mathbf{S}_{2, i+\nu}\right)\right]
\end{aligned}
$$

contains the anisotropic symmetric exchange interaction, which is given in terms of an energy $J$ and dimensionless factors $\gamma$ and $\lambda$, and the antisymmetric exchange interaction described by DMI vectors along $z$ direction. We choose $\gamma \leqslant 1$ and $\lambda \geqslant 1$, such that the collinear state with Néel vector along $z$ direction is the classical magnetic ground state. For $\gamma \neq 1$, the anisotropy causes the magnons to experience the effect of "squeezing" [71]. Note that $\lambda$ has to be larger than a critical value to avoid the spins from canting due to DMI. The DMI strength is set to $D_{12}^{+}=D_{1}$ and $D_{12}^{-}=D_{2}$, where $v= \pm$ refers to the direction of the bond $[+$ for going from the left to the right in Fig. 1(a)].

It is convenient to reparametrize the DMI as $D_{0}=\left(D_{1}+\right.$ $\left.D_{2}\right) / 2 J$ and $\delta_{D}=\left(D_{1}-D_{2}\right) / 2 J$. The staggered contribution to DMI is necessary for the model to exhibit both intrinsic as well as extrinsic effects. To see this, observe that only in the absence of the inversion symmetry we can have $D_{0} \neq 0$. However, when $\delta_{D}=0$, the system still holds a $\mathcal{T} * \mathcal{M}_{x}$ symmetry, where $\mathcal{T}$ is time reversal and $\mathcal{M}_{x}$ is the mirror symmetry with respect to the $y-z$ plane passing through the atoms. Applying the corresponding Cartesian representation matrix $R=\operatorname{Diag}\{-1,1,1\}$ of $\mathcal{T} * \mathcal{M}_{x}$ to Eq. (36), the intrinsic part (a)
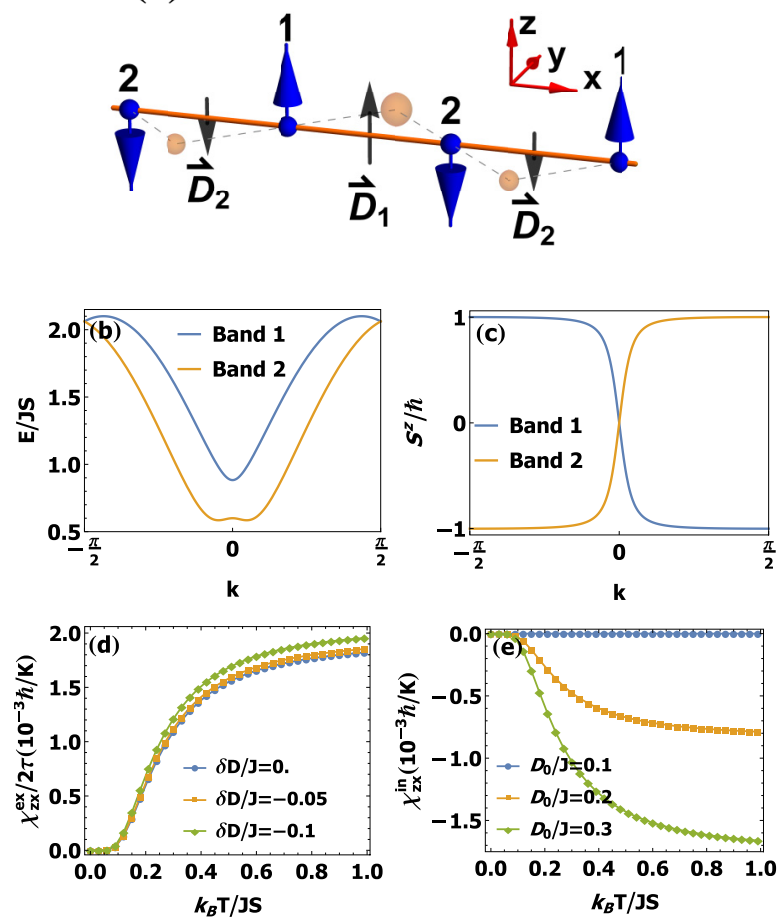

FIG. 1. (a) Spin order and DMI vectors in the antiferromagnet spin chain model. (b), (c) Magnon dispersion and magnon spin expectation value in the one-dimensional (1D) Brillouin zone. We used $D_{0} / J=0.2, \delta D / J=-0.1$. (d), (e) Extrinsic and intrinsic response coefficients. In (d), $\tau=J S /\left(2 \Gamma_{n}\right)$ is the dimensionless magnon lifetime ( $\hbar$ is set to 1 ). Parameters read $\lambda=1.05, \gamma=0.95$, $J=2 \mathrm{meV}, S=3 / 2$, and $D_{0} / J=0.2$.

$\chi_{z x}^{\text {in }}$ is rendered zero. Therefore, we set $\delta_{D} \neq 0$ to ensure the appearance of intrinsic contributions.

In Fig. 1(b), we show the magnon band structure. The degeneracy of spin-up and -down modes is lifted by the DMI and $\gamma \neq 1$. On top of that, since $\gamma \neq 1$, spin is not conserved and we observe the magnon spin-momentum locking [27] as shown in Fig. 1(c), which is in agreement with Ref. [71]. This is in contrast to the usual case of uniaxial collinear antiferromagnets (AFMs) that feature two eigenmodes with opposite spin quanta $\pm \hbar$. Figures 1 (d) and 1(e) show the extrinsic and intrinsic response coefficient, respectively. For the calculation of the extrinsic response, we regarded the broadening as a constant, $\Gamma_{n}=\hbar / 2 \tau$, where $\tau$ is the magnon lifetime [72]. In Figs. 1(d) and 1(e), the extrinsic spin accumulation dominates.

To obtain an intuitive understanding of the extrinsic contributions, we recall the usual electronic Edelstein effect scenario in a Rashba system. Upon shifting the spin-momentum locked Fermi circles in reciprocal space due to application of an electric field, electronic states with a particular spin polarization are more occupied than those with opposite spin polarization (e.g., see Fig. 13 of Ref. [73]). Consequently, this redistribution leads to a nonzero macroscopic spin density in nonequilibrium. A similar explanation can be given for the magnonic case. First, we consider the band 2 [cf. Fig. 1(b)]. According to Fig. 1(c), magnons in band 2 have a positive (negative) spin for negative (positive) momentum $k$, which 


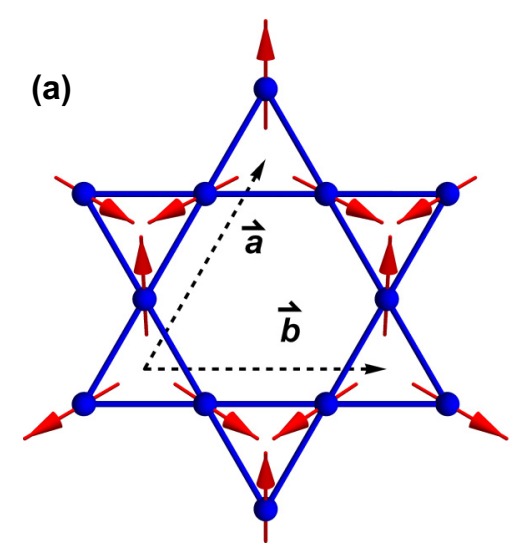

(b)

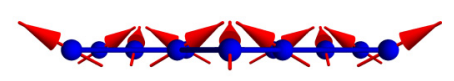

(c)

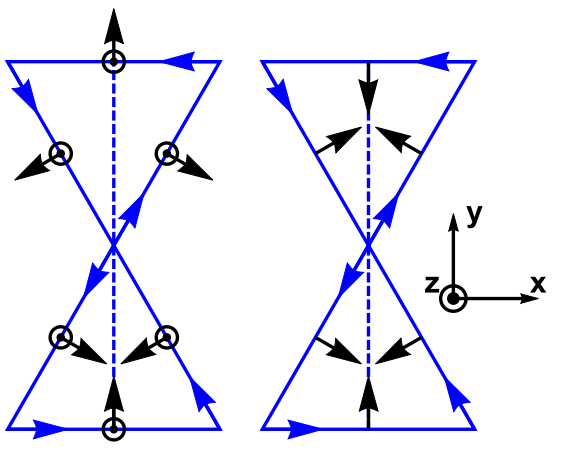

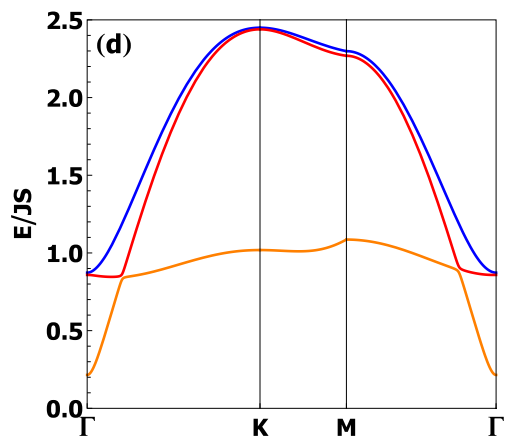
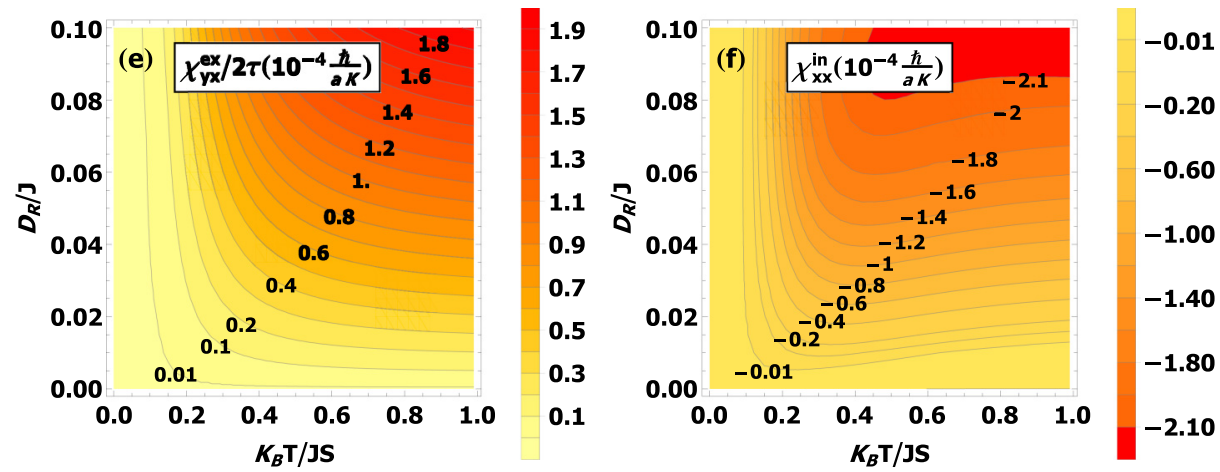

FIG. 2. Noncollinear antiferromagnetic PVC order on the kagome lattice. (a), (b) Ground-state spin configuration from above and front view. Lattice vectors are denoted by $\vec{a}$ and $\vec{b}$. (c) Left: intrinsic DMI vectors; right: Rashba DMI vectors. Arrows along the bonds indicate ordering of sites in DMI terms. (d) Magnon dispersion with $D_{R} / J=0.06$. (e), (f) Extrinsic and intrinsic response tensor elements $\chi_{y x}^{\text {ex }}$ and $\chi_{x x}^{\text {in }}$, respectively. $\tau$ is the dimensionless magnon lifetime and $a$ denotes the lattice constant. We used the material parameters of KFe $3(\mathrm{OH})_{6}\left(\mathrm{SO}_{4}\right)_{2}$ : $J_{1}=3.18 \mathrm{meV}, J_{2}=0.11 \mathrm{meV},\left|D_{p}\right| / J_{1}=0.062, D_{z} / J_{1}=-0.062$, and $S=5 / 2$.

corresponds to magnon spin-momentum locking discussed in Ref. [27]. Upon application of the temperature gradient (or the pseudogravitational potential) we redistribute magnons from $k$ to $-k$ (or vice versa, depending on the direction of the gradient), causing an excess of magnons with positive spin. Although there is some cancellation between the lower and upper band, the different thermal occupation ensures that there is a nonzero resulting net spin density in nonequilibrium. There is no such simple picture for the intrinsic contributions, which arise due to interband mixing [2].

\section{B. Kagome antiferromagnet}

In several real materials, spin nonconservation naturally emerges due to noncollinear antiferromagnetism. For example, noncollinear antiferromagnets (NAFMs) exist in layered quasi-two-dimensional kagome and triangular magnetic structures, and in three-dimensional pyrochlore magnetic structures. We first take the kagome antiferromagnet in the so-called $\mathbf{q}=0$ phase with positive vector chirality (PVC) $[62,74,75]$, which is depicted in Fig. 2(a), as an example.

The spin Hamiltonian under consideration is

$$
H=\sum_{\langle i j\rangle} J_{1} \mathbf{S}_{i} \cdot \mathbf{S}_{j}+\mathbf{D}_{i j} \cdot\left(\mathbf{S}_{i} \times \mathbf{S}_{j}\right)+\sum_{\langle\langle i j\rangle\rangle} J_{2} \mathbf{S}_{i} \cdot \mathbf{S}_{j},
$$

where the three terms describe the nearest-neighbor exchange with $J_{1}>0$, DMI, and the second-nearest neighbor exchange with $J_{2}>0$. The DMI vector $\mathbf{D}_{i j}$ is composed of intrinsic DMI and extrinsic Rashba DMI, i.e., $\mathbf{D}_{i j}=\mathbf{D}_{\text {in }}+\mathbf{D}_{R}$. The intrinsic DMI $\mathbf{D}_{\text {in }}=\mathbf{D}_{p}+D_{z, i j} \hat{z}$ has out-of-plane contributions $D_{z, i j}$ as well as in-plane contributions $\mathbf{D}_{p}=D_{p} \hat{n}_{i j}$ along $\hat{n}_{i j}$. The DMI vectors are arranged as shown in the left part of Fig. 2(c). Accounting for the antiferromagnetic exchange interactions and only for the intrinsic DMI, the classical ground state is the $120^{\circ}$-ordered antiferromagnetic state [cf. Fig. 2(a)] with a small out-of-plane canting, with an angle given by $\eta=\frac{1}{2} \tan ^{-1}\left(\frac{-2 D_{p}}{\sqrt{3\left(J_{1}+J_{2}\right)}-D_{z}}\right)$ [cf. Fig. 2(b)]. Thus, there is a weak ferromagnetic moment in the $z$ direction and the texture is not fully compensated. Here, we are concentrating on nonequilibrium spin densities in the $x$ and $y$ directions, along which the texture is compensated.

Although nonzero $\mathbf{D}_{p}$ breaks the mirror symmetry of the kagome lattice, the system is still inversion symmetric. Thus, we need the Rashba-like DMI described by $\mathbf{D}_{R}$ that we envision to arise in an inversion-symmetry breaking environment, as caused, e.g., by putting a single kagome layer on a substrate. The vector $\mathbf{D}_{R}$ lies in the kagome plane and has directions similar to $\mathbf{D}_{p}$, but with the crucial difference that its directions are always pointing in the same direction relative to the bond [compare the left and right parts of Fig. 2(c)]. 
TABLE I. The shape of spin-polarization response tensors enforced by magnetic point-group symmetry for selected noncollinear antiferromagnets.

\begin{tabular}{lcc}
\hline \hline Structure & Extrinsic & Intrinsic \\
\hline Kagome (PVC,SVC) & $\left(\begin{array}{cc}0 & -\chi_{y x}^{\mathrm{ex}} \\
\chi_{y x}^{\mathrm{ex}} & 0\end{array}\right)$ & $\left(\begin{array}{cc}\chi_{x x}^{\mathrm{in}} & 0 \\
0 & \chi_{x x}^{\mathrm{in}}\end{array}\right)$ \\
Kagome (NVC) & $\left(\begin{array}{cc}0 & \chi_{x y}^{\mathrm{ex}} \\
\chi_{y x}^{\mathrm{ex}} & 0\end{array}\right)$ & $\left(\begin{array}{cc}\chi_{x x}^{\mathrm{in}} & 0 \\
0 & \chi_{y y}^{\mathrm{in}}\end{array}\right)$ \\
Triangle & $\left(\begin{array}{cc}0 & -\chi_{y x}^{\mathrm{ex}} \\
\chi_{y x}^{\mathrm{ex}} & 0\end{array}\right)$ & $\left(\begin{array}{cc}\chi_{x x}^{\mathrm{in}} & 0 \\
0 & \chi_{x x}^{\mathrm{in}}\end{array}\right)$ \\
Pyrochlore (AIAO) & $\left(\begin{array}{ccc}0 & 0 & 0 \\
0 & 0 & 0 \\
0 & 0 & 0\end{array}\right)$ & $\left(\begin{array}{ccc}\chi_{x x}^{\mathrm{in}} & 0 & 0 \\
0 & \chi_{x x}^{\mathrm{in}} & 0 \\
0 & 0 & \chi_{x x}^{\mathrm{in}}\end{array}\right)$ \\
\hline \hline
\end{tabular}

We also note that a large Rashba-DMI can twist the system into a spiral state. We confirmed numerically that this does not happen for $\left|\mathbf{D}_{R}\right| / J<0.06$ using computational package SpinW [76].

The kagome NAFM described above exhibits two symmetries: (i) the mirror reflection with respect to the $y-z$ plane plus time reversal, $g_{1}=\mathcal{M}_{x} \mathcal{T}$, and (ii) the threefold rotation about the $z$ axis, $g_{2}=\mathcal{C}_{3 z}$. According to Eq. (36), these two symmetries fix the extrinsic and intrinsic response tensors to the forms in Table I (kagome PVC), where only the in-plane spin polarizations are allowed.

Based on what we have discussed so far, we propose potassium iron jarosite $\mathrm{KFe}_{3}(\mathrm{OH})_{6}\left(\mathrm{SO}_{4}\right)_{2}$ as a candidate material. Concentrating on a single kagome layer of this material and assuming that the mirror symmetry is broken due to a proper environment, the magnon dispersion is given in Fig. 2(d). We used the parameters $J_{1}=3.18 \mathrm{meV}, J_{2}=$ $0.11 \mathrm{meV},\left|D_{p}\right| / J_{1}=0.062, D_{z} / J_{1}=-0.062$, and $S=5 / 2$ $[36,77]$. The spin-density response is captured by virtue of Eqs. (21) and (35). The results for the extrinsic $\chi_{y x}^{\mathrm{ex}}$ and intrinsic contributions, $\chi_{x x}^{\text {in }}$, are shown in Figs. 2(e) and 2(f), respectively. The effect becomes stronger as we increase Rashba DMI. The contributions $\chi_{x x}^{\text {ex }}$ and $\chi_{y x}^{\text {in }}$ are zero in agreement with tensor shapes in Table I.

Approximating the magnon band broadening $\Gamma_{n} \sim \hbar / 2 \tau$ as a constant, with a magnon lifetime $\tau \sim 10^{-10} \mathrm{~s}$, and using a lattice constant $a=10^{-9} \mathrm{~m}$, a Rashba DMI $D_{R}=$ $0.06 \mathrm{~J}$, a temperature gradient $\partial_{x} T=10 \mathrm{~K} / \mathrm{mm}$ [78], and a temperature $T=0.5 \mathrm{JS}$ [which corresponds to a temperature $\approx 46 \mathrm{~K}$ for $\left.\mathrm{KFe}_{3}(\mathrm{OH})_{6}\left(\mathrm{SO}_{4}\right)_{2}\right]$, we obtain the extrinsic part of the temperature-gradient-induced spin density $\left\langle\Sigma_{y}\right\rangle_{\mathrm{ex}} \simeq$ $5 \times 10^{6} \hbar / \mathrm{cm}^{2}$, and the intrinsic part $\left\langle\Sigma_{x}\right\rangle_{\text {in }} \simeq 2 \times 10^{5} \hbar / \mathrm{cm}^{2}$. With larger temperature gradients, the extrinsic contribution can be made comparable to spin densities generated by the electronic Edelstein effect [14], which are of the order of $10^{8}-10^{10} \hbar / \mathrm{cm}^{2}$.

\section{Breathing pyrochlore antiferromagnets}

The 3D pyrochlore lattices, which consist of cornersharing tetrahedra, are well known for exhibiting noncollinear spin structures. Here, to break bulk inversion symmetry, we concentrate on the so-called "breathing" pyrochlore (a)
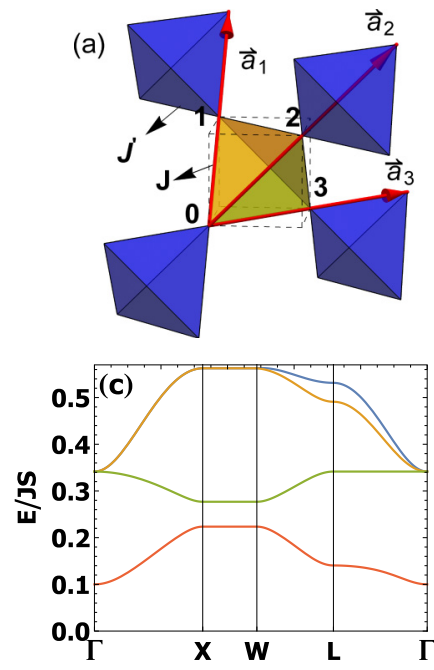

(b)
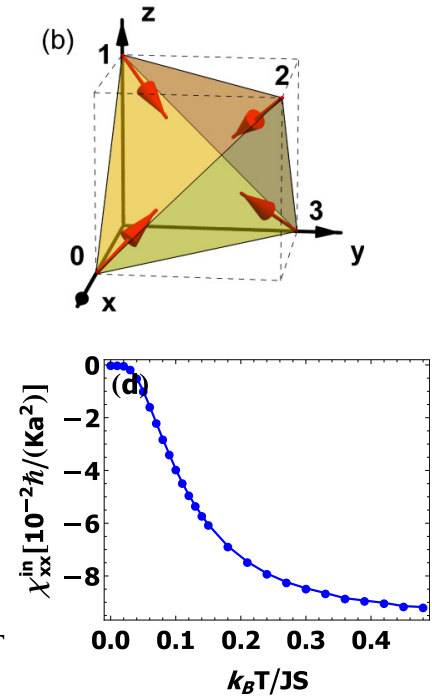

FIG. 3. (a) Breathing pyrochlore lattice with indicated lattice vectors $\vec{a}_{i}(i=1,2,3)$ and nearest-neighbor exchange in up-pointing (blue, $J^{\prime}$ ) and down-pointing (yellow, $J$ ) tetrahedra. (b) Spin order in the all-in-all-out configuration. (c) Magnon band structure. (d) The intrinsic response $\chi_{x x}^{\text {in }}$, with $a$ denoting the lattice constant. Parameters read $J \approx 50 \mathrm{~K}(4.3 \mathrm{meV}), J^{\prime} / J=0.6, D / J=-0.2, S=3 / 2$ to mimic the material $\mathrm{LiGaCr}_{4} \mathrm{O}_{8}$.

antiferromagnets that possess different exchange interaction in up-pointing $(u)$ and down-pointing $(d)$ tetrahedra [see Fig. 3(a)]. The minimal Heisenberg model is [79]

$$
H=J \sum_{\langle i, j\rangle \in u} \mathbf{S}_{i} \cdot \mathbf{S}_{j}+J^{\prime} \sum_{\langle i, j\rangle \in d} \mathbf{S}_{i} \cdot \mathbf{S}_{j}+D \sum_{i}\left(\mathbf{S}_{i} \cdot \hat{\mathbf{z}}_{i}\right)^{2} .
$$

The first two terms describe the antiferromagnetic exchange interactions in up-pointing and down-pointing tetrahedra, respectively. The last term comprises easy-axis anisotropy $(D<$ 0 ), with $\hat{\mathbf{z}}_{i}$ being a unit vector pointing either towards or away from the tetrahedon's center of gravity. This model can be energetically optimized to different spin configurations depending on the values of $J^{\prime} / J$, and $D / J[79,80]$, but here we only concentrate on the all-in-all-out (AIAO) order depicted in Fig. 3(b), in which all spins of a single tetrahedron are either pointing inward [yellow tetrahedron in Fig. 3(b)] or outward (blue tetrahedra).

The AIAO order respects the magnetic point group $\bar{T}_{d}=$ $\operatorname{Span}\left\{C_{3}, C_{2}, \mathcal{T} * \sigma_{d}, \mathcal{T} * S_{4}\right\}[81,82]$. Here, we give the representative generators of these symmetries: $C_{3}$ is the threefold rotation with respect to the $[1,1,1]$ axis; $C_{2}$ is the twofold rotation about the $[1,0,0]$ axis; $\mathcal{T} * \sigma_{d}$ is time reversal followed by the reflection about the $(\overline{1}, 1,0)$ plane; and $\mathcal{T} * S_{4}$ is time reversal followed by the combination of the fourfold rotation about $[1,0,0]$ and the reflection about $(1,0,0)$. We find that this symmetry constraint eliminates any extrinsic response and enforces the intrinsic response tensor to be proportional to a unit matrix; see Table I. In Fig. 3(c), we plot the dispersion of the four magnon bands for the AIAO phase with $J \approx 50 \mathrm{~K}(4.3 \mathrm{meV})$ and $J^{\prime} / J=0.6$, which is the breathing ratio of $\mathrm{LiGaCr}_{4} \mathrm{O}_{8}$ [83]. We used $D / J=-0.2$ to stabilize the AIAO order. In Fig. 3(d), we show the intrinsic response $\chi_{x x}^{\text {in }}=\chi_{y y}^{\text {in }}=\chi_{z z}^{\text {in }}$, which are the only nonzero tensor 
elements, in agreement with the symmetry analysis. If we assume $\partial_{x} T=10 \mathrm{~K} / \mathrm{mm}, T=0.12 J S$, and $a \sim 10^{-9} \mathrm{~m}$, the intrinsic spin accumulation is estimated to be $\left\langle\Sigma_{x}\right\rangle_{\text {in }} \simeq 5 \times$ $10^{14} \hbar / \mathrm{cm}^{3}$. We can compare this result with the electronic Edelstein effect by converting its $2 \mathrm{D}$ spin density to a bulk density: $\langle\Sigma\rangle_{\text {electron }}^{2 \mathrm{D}} / a \sim 10^{15}-10^{17} \hbar / \mathrm{cm}^{3}$. Thus, the intrinsic contribution in breathing pyrochlores is comparable with the electronic Edelstein effect. We believe that this result is detectable in experiment either by transport measurements similar to those used for detection of the inverse spin Hall effect, by magneto-optical Kerr microscopy, or by magnetic sensing based on the nitrogen-vacancy $(N V)$ centers [84].

\section{COMPUTER EXPERIMENTS}

To better understand the nonequilibrium spin density brought about by the magnonic counterpart to the Edelstein effect, we use atomistic spin dynamics simulations. We describe spin dynamics using the stochastic Landau-Lifshitz-Gilbert (sLLG) equation

$$
\dot{\mathbf{S}}_{i}=-\frac{\gamma}{\mu\left(1+\alpha^{2}\right)}\left[\mathbf{S}_{i} \times \mathbf{B}_{i}+\alpha \mathbf{S}_{i} \times\left(\mathbf{S}_{i} \times \mathbf{B}_{i}\right)\right],
$$

comprising the damped precession of $\mathbf{S}_{i}$ about its local field $\mathbf{B}_{i}=\mathbf{b}_{i}-\partial H / \partial \mathbf{S}_{i}$. The stochastic field $\mathbf{b}_{i}(t)=$ $\sqrt{2 \alpha k_{\mathrm{B}} T \mu /(\gamma \Delta t)} \mathbf{G}(t)$ simulates thermal noise $[85,86] . \mathbf{G}(t)$ is a three-dimensional Gaussian random number distribution with zero mean. $\alpha, \gamma$, and $\mu=2 \mu_{\mathrm{B}} \sqrt{S(S+1)}$ are the dimensionless Gilbert damping, the gyromagnetic ratio, and the modulus of the magnetic moment at each lattice site, respectively. The numerical integration of Eq. (41) is done by the Heun method [86] with time steps $\Delta t \leqslant 1$ fs.

We consider the antiferromagnetic spin chain introduced in Sec. V A and study this model in a nonequilibrium situation. As was shown in Sec. VA, the extrinsic contribution to the nonequilibrium spin density dominates over the intrinsic contribution for the spin chain model. Thus, we focus on the extrinsic contributions and set $D_{1}=D_{2}=D$ such that $\delta_{D}=0$, rendering intrinsic contributions zero by symmetry.

We simulate a spin chain of $N=480$ spins with spin Hamiltonian as in Eq. (38). First, to characterize the chain in terms of magnon variables, i.e., in terms of (i) the magnon dispersion and (ii) the magnon spin, we calculate the dynamical structure factor

$\mathcal{F}(k, \omega)=\frac{1}{\sqrt{2 \pi} N} \sum_{i, j} e^{i k\left(x_{i}-x_{j}\right)} \int_{-\infty}^{\infty} e^{i \omega t}\left\langle S_{i}^{+}(t) S_{j}^{-}(0)\right\rangle d t$,

i.e., the time and space Fourier transform of the spin-spin time-correlation function. $x_{i}$ denotes the $x$ coordinate of the $i$ th spin and $S_{i}^{ \pm}=S_{i}^{x} \pm i S_{i}^{y}$.

The numerically determined magnon spectra for the spin chain are shown in Figs. 4(a), 4(c) 4(e), and 4(g); they agree with those obtained analytically in the previous section [shown as black lines in Figs. 4(b), 4(d) 4(f), and 4(h)]. In Fig. 4(a), we depict the dispersion of the isotropic antiferromagnetic spin chain $(\lambda=1, D=0, \gamma=1)$ with the two degenerate linear Goldstone modes. This degeneracy is lifted in the presence of spin-nonconserving anisotropies $\lambda>1$ and $\gamma<1$ [cf. Fig. 4(c)]. In Fig. 4(e), we show the Rashba-like spin-split dispersion in the presence of nonzero DMI and $\lambda>1$, and in Fig. 4(g) the dispersion in the presence of both
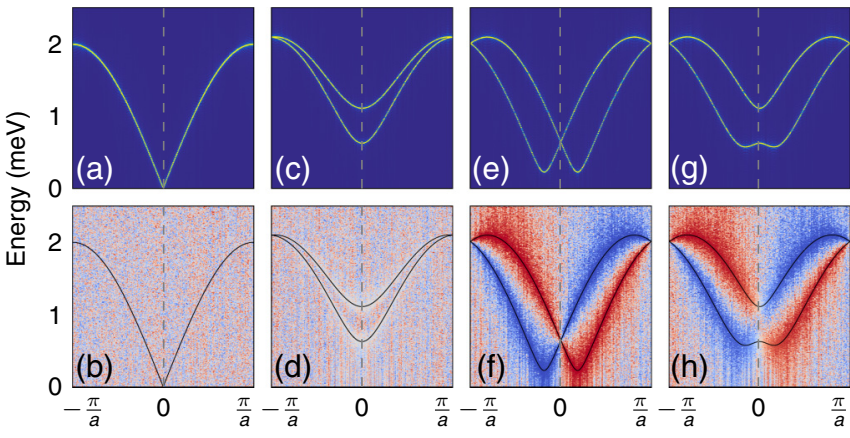

FIG. 4. Magnon spectra of the antiferromagnetic spin chain as obtained from numerical simulations for selected parameters. Top row: dynamical structure factor; bottom row: the spin of magnons or Stokes parameter ratio $\sigma(k, \omega)$ (red: negative; gray: zero; blue: positive). Black solid lines show the analytically obtained magnon dispersion (within linear spin-wave theory). Parameters read $J=$ $1 \mathrm{meV}$, and (a), (b) $\lambda=1, D=0, \gamma=1$, (c), (d) $\lambda=1.05, D=$ $0, \gamma=0.9$, (e), (f) $\lambda=1.05, D=0.3 \mathrm{meV}, \gamma=1$, and (g), (h) $\lambda=1.05, D=0.3 \mathrm{meV}, \gamma=0.9$. A small simulation temperature $T=0.01 \mathrm{~K}$ and Gilbert damping $\alpha=0.001$ were chosen to reduce lifetime broadening.

anisotropies as well as DMI, for which the band degeneracy at $k=0$ is lifted [as compared to Fig. 4(e)].

The magnon spin is extracted by computing the Stokes parameters $I(k, \omega)=\left|\mathcal{S}^{x}\right|^{2}+\left|\mathcal{S}^{y}\right|^{2}$ and $V(k, \omega)=$ $-2 \operatorname{Im}\left(\mathcal{S}^{x} \mathcal{S}^{y, *}\right)$ [87], where $\mathcal{S}=\mathcal{S}(k, \omega)$ is the space and time Fourier transform of the spin configuration $\left\{\mathbf{S}_{i}(t)\right\}$. The quantity $\sigma(k, \omega)=V(k, \omega) / I(k, \omega)$ measures the ratio of circular to total polarization intensity; its sign reveals the sign of the magnon spin. There is no feature of $\sigma(k, \omega)$ in Fig. 4(b), in agreement with the previous section. In contrast, $\sigma(k, \omega)$ becomes zero (gray color) in Fig. 4(d), indicating that the magnon spin is suppressed due to ellipticity or "squeezing," which is in agreement with Ref. [71]. Without squeezing but nonzero DMI we identify spin-up and spin-down magnons by the antisymmetric blue-red features in Fig. 4(f). In the presence of squeezing and DMI this asymmetric feature survives [panel (h)] and shows that the spin expectation value continuously goes through zero upon crossing $k=0$, an observation which is in agreement with Fig. 1(c).

In the previous section, we obtained a nonzero magnonic spin polarization for the case in Figs. 4(g) and 4(h) [which are respectively reminiscent of Figs. 1(b) and 1(c)], but zero effect for the other cases. We will now put this prediction to the test

To do so, direct nonequilibrium simulations with an imprinted temperature gradient were performed. The spin chain was separated into three parts of equal length (160 spins each). The terminating parts have temperature $T \pm \Delta T / 2$, while the temperature in the central part linearly interpolates between the two ends. Following this temperature profile, a heat bath with temperature $T_{i}$ is assigned to each spin $i$. After establishing a steady state in this nonequilibrium situation, the spin density $\langle\mathbf{S}\rangle=\frac{1}{160} \sum_{i=161}^{320}\left\langle\mathbf{S}_{i}\right\rangle$ of the central chain segment is measured and normalized to the number of spins in this segment. (This nonequilibrium averaged $\langle\mathbf{S}\rangle$ takes over the role of $\langle\boldsymbol{\Sigma}\rangle$.) 


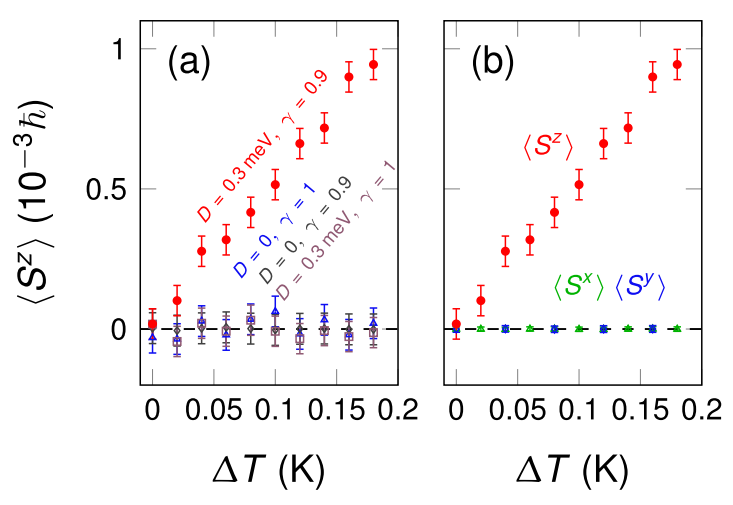

FIG. 5. Results from direct nonequilibrium spin dynamics simulations of the thermally induced magnonic analog of the Edelstein effect in an anisotropic antiferromagnetic spin chain; parameters read $J=1 \mathrm{meV}, \lambda=1.02$, and $\alpha=10^{-4}$. (a) Nonequilibrium spin density $\left\langle S^{z}\right\rangle$ in dependence on temperature difference $\Delta T$ for selected parameter combinations. (b) $\left\langle S^{i}\right\rangle(i=x, y, z)$ in dependence on $\Delta T$. An average temperature of $T=0.2 \mathrm{~K}$ was used for all simulations.

There is an additional technicality of the simulation: Since two neighboring spins in the central chain segment experience slightly different temperatures $\left(T_{i} \neq T_{i+1}\right)$, their net moment does not compensate exactly. Repeating this argument for all spins of the central segment, we conclude that there is a tiny net magnetization simply due to the temperature dependence of the sublattice magnetizations. The sign of this artificial magnetization is determined by the direction of the first spin at the cold end of the central segment. This artificial effect would superimpose with the magnon analog of the Edelstein effect. Thus, to avoid the non-Edelstein contribution, we simulate two uncoupled spin chains with opposite spin textures in parallel. The non-Edelstein contributions are exactly opposite, because the sublattice magnetization is reversed, and sum to zero. In contrast, the extrinsic Edelstein contributions are time reversal even as shown in Eq. (37b) and do not cancel out.

Our simulation results are presented in Fig. 5. The $z$ spin accumulation $\left\langle S^{z}\right\rangle$ is zero in equilibrium $[\Delta T=0$ in Fig. 5(a)], as expected for an antiferromagnet in zero magnetic field. It stays zero in nonequilibrium $(\Delta T \neq 0)$, if either DMI or squeezing (or both) are absent [compare brown, blue, and purple marks in Fig. 5(a)]. However, it becomes nonzero if DMI and squeezing are present (red marks), in full agreement with theory.

The other Cartesian components of the spin density, i.e., $\left\langle S^{x}\right\rangle$ and $\left\langle S^{y}\right\rangle$, are zero even in nonequilibrium [blue and green marks in Fig. 5(b)]. This is not surprising, because no magnon state has a nonzero $x$ or $y$ spin. Thus, a nonequilibrium state cannot give rise to spin density of those components. In contrast, $\left\langle S^{z}\right\rangle$ increases approximately linearly with the external force $\Delta T$.

We note in passing other results that are not explicitly shown. We found that (i) reversing $D$ reverses $\left\langle S^{z}\right\rangle$ due to the reversion of the magnon spin, (ii) increasing $\lambda$ increases the magnon gap, leading to a decreasing $\left\langle S^{z}\right\rangle$, and (iii) increasing the Gilbert damping $\alpha$ diminishes $\left\langle S^{z}\right\rangle$, because the magnon transport lifetime decreases.

Overall, we find excellent qualitative agreement with theory (Sec. V A). However, we mention that we cannot compare
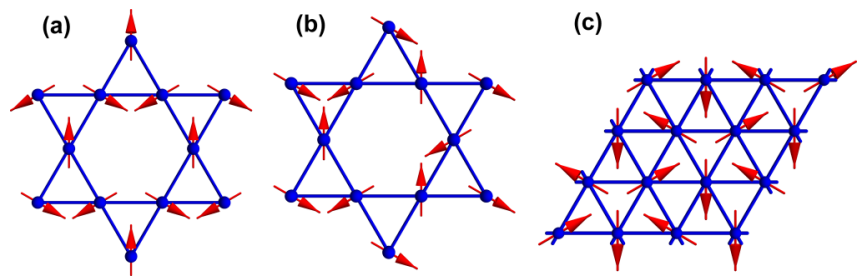

FIG. 6. (a), (b) Noncollinear spin textures on the kagome lattice, with (a) negative vector chirality (NVC) and (b) staggered vector chirality (SVC). (c) Noncollinear antiferromagnetic ground state on the 2D triangular lattice.

numbers, because the classical white noise used to model the temperature bath results in a Rayleigh-Jeans distribution rather than in the true Bose-Einstein distribution. Thus, the simulation suffers from the classical equipartition and does not account for the quantum freezing of degrees of freedom as temperature goes to zero.

\section{CONCLUSION}

We have shown that a temperature gradient can induce a nonequilibrium spin density due to magnonic transport in antiferromagnetic insulators with inversion asymmetry and spin nonconservation. Our linear-response theory revealed both intrinsic and extrinsic contributions that behave differently under time reversal. Consequently, these two contributions correspond to different elements of the response tensor, which can facilitate their experimental disentanglement, e.g., in the presence of magnetic domains. Our proposal can be realized in (quasi-)2D and 3D noncollinear antiferromagnets, for which we find sizable effects in realistic material candidates. Our predictions can be tested by transport measurements similar to those used for detection of the inverse spin Hall effect, by magneto-optical Kerr microscopy, or by magnetic sensing based on the nitrogen-vacancy $(N V)$ centers. Given the omnipresence of inversion-symmetry-breaking interfaces (or surfaces) in experimental setups, observation of the magnonic analog of the Edelstein effect can stimulate further developments in the field of spintronics. In particular, with the important role played by the electronic Edelstein effect in modern spintronics in mind, we hope to have stimulated experimental research on the magnonic analog of the Edelstein effect.

The abundance of antiferromagnetic materials holds great promise for the identification of well-suited experimental candidates. In kagome noncollinear antiferromagnets, the coplanar magnetic order can exhibit three types of vector chiralities: positive, negative, and staggered, which are respectively abbreviated by PVC, NVC, and SVC [75,88] and depicted in Figs. 2(a), 6(a), and 6(b). Their distinct magnetic symmetries cause distinct magnonic spin polarization responses, which are tabulated in Table I. Besides kagome magnets, quasi-2D triangular antiferromagnets [cf. Fig. 6(c)] with the $120^{\circ}$ spin order $[89,90]$ could be suitable candidates. Such systems as $\mathrm{RbFe}\left(\mathrm{MoO}_{4}\right)_{2}$ [91] and $\mathrm{Ba}_{3} \mathrm{NiNb}_{2} \mathrm{O}_{9}$ [92] share symmetries with the PVC kagome noncollinear antiferromagnets, resulting in identical response tensor shapes (cf. Table I). Similar to kagome noncollinear antiferromagnets, the 3D breathing pyrochlores can exhibit magnetic orders different 
from the all-in-all-out order [79,80], which changes their magnetic symmetries and, thus, the expected response tensor shapes. Experimentally, the breathing pyrochlore materials $\mathrm{Ba}_{3} \mathrm{Yb}_{2} \mathrm{Zn}_{5} \mathrm{O}_{11}$ [93,94], $\mathrm{LiInCr}_{4} \mathrm{O}_{8}$ [95] have been studied, all of which may be considered for a proof-of-principle study of our predictions.

Note added. Recently, we noticed that Ref. [96] discussed the intrinsic magnon spin polarization for a compensated ferrimagnet with different $g$ factors for two magnetic sublattices but did not consider Dzyaloshinskii-Moriya interactions.

\section{ACKNOWLEDGMENTS}

We thank S. Sandhoefner for helpful discussions. This work was supported by DOE Early Career Award No. DESC0014189.

\section{APPENDIX A: LINEAR RESPONSE FOR ANTIFERROMAGNETS}

\section{General theory}

For the $\mu$ component of a spatially averaged observable $A_{\mu}=\frac{1}{V} \int d \mathbf{r} \Psi^{\dagger}(\mathbf{r}) \hat{A}_{\mu} \Psi(\mathbf{r})$, the nonequilibrium response to a temperature gradient is

$$
\left\langle A_{\mu}\right\rangle_{\mathrm{ne}}=\lim _{\omega \rightarrow 0} \frac{1}{i \omega}\left[\Pi_{\mu \nu}(\omega)-\Pi_{\mu \nu}(0)\right] \nabla_{\nu} \phi
$$

where the correlator in frequency space is defined as

$$
\Pi_{\mu \nu}\left(i \omega_{m}\right)=-\int_{0}^{\beta} d \tau e^{i \omega_{m} \tau}\left\langle T_{\tau} A_{\mu}(\tau) J_{\nu}^{q}(0)\right\rangle
$$

In momentum space, $A_{\mu}=\frac{1}{V} \sum_{\mathbf{k}} \Psi_{\mathbf{k}}^{\dagger} A_{\mu, \mathbf{k}} \Psi_{\mathbf{k}}$ and $J_{v}^{q}=$ $\sum_{\mathbf{k}} \Psi_{\mathbf{k}}^{\dagger} J_{v, \mathbf{k}}^{q} \Psi_{\mathbf{k}}$, with $J_{v, \mathbf{k}}^{q}=\frac{1}{4}\left(\mathcal{H}_{\mathbf{k}} \sigma_{3} \mathbf{v}_{v, \mathbf{k}}+\mathbf{v}_{v, \mathbf{k}} \sigma_{3} \mathcal{H}_{\mathbf{k}}\right)$. Here, $J_{v}^{q}$ comes from $\frac{\partial H^{\prime}}{\partial t}=\frac{i}{\hbar}\left[H, H^{\prime}\right]=J_{\nu}^{q} \nabla_{\nu} \phi$; see the Supplemental Material of Refs. [29,43]. Plugging in the above expressions, the correlation tensor can be presented as

$$
\begin{aligned}
\Pi_{\mu \nu}\left(i \omega_{m}\right) & =-\frac{1}{V} \sum_{\mathbf{k}, \mathbf{k}^{\prime}} \int_{0}^{\beta} d \tau e^{i \omega_{m} \tau}\left\langle\Psi_{\mathbf{k}}^{\dagger}(\tau) A_{\mu, \mathbf{k}} \Psi_{\mathbf{k}}(\tau) \Psi_{\mathbf{k}^{\prime}}^{\dagger} J_{\nu, \mathbf{k}^{\prime}}^{q} \Psi_{\mathbf{k}^{\prime}}\right\rangle \\
& =-\frac{1}{V} \sum_{\mathbf{k}, \mathbf{k}^{\prime}} \int_{0}^{\beta} d \tau e^{i \omega_{m} \tau}\left(A_{\mu, \mathbf{k}}\right)_{\alpha \gamma}\left(J_{\nu, \mathbf{k} /}^{q}\right)_{\rho \sigma}\left\langle T_{\tau} \Psi_{\mathbf{k}, \alpha}^{\dagger}(\tau) \Psi_{\mathbf{k}, \gamma}(\tau) \Psi_{\mathbf{k}^{\prime}, \rho}^{\dagger}(0) \Psi_{\mathbf{k}^{\prime}, \sigma}(0)\right\rangle
\end{aligned}
$$

According to Wick's theorem,

$$
\begin{aligned}
\left\langle T_{\tau} \Psi_{\mathbf{k}, \alpha}^{\dagger}(\tau) \Psi_{\mathbf{k}, \gamma}(\tau) \Psi_{\mathbf{k}^{\prime}, \rho}^{\dagger}(0) \Psi_{\mathbf{k}^{\prime}, \sigma}(0)\right\rangle_{\text {connected }}= & \left\langle T_{\tau} \Psi_{\mathbf{k}^{\prime}, \sigma}(0) \Psi_{\mathbf{k}, \alpha}^{\dagger}(\tau)\right\rangle\left\langle T_{\tau} \Psi_{\mathbf{k}, \gamma}(\tau) \Psi_{\mathbf{k}, \rho}^{\dagger}(0)\right\rangle \\
& +\left\langle T_{\tau} \Psi_{\mathbf{k}, \alpha}^{\dagger}(\tau) \Psi_{\mathbf{k}, \rho}^{\dagger}(0)\right\rangle\left\langle T_{\tau} \Psi_{\mathbf{k}^{\prime}, \gamma}(\tau) \Psi_{\mathbf{k}, \sigma}(0)\right\rangle
\end{aligned}
$$

Here, the second anomalous term can be shown to be equivalent to the first term. First, we note that the basis $\Psi_{\mathbf{k}}$ obeys the particle-hole symmetry, $\Psi_{\mathbf{k}}=\left(\Psi_{-\mathbf{k}}^{\dagger} \sigma_{1}\right)^{T}$, which leads to the relation

$$
A_{\mu}=\frac{1}{V} \sum_{\mathbf{k}, \alpha \beta} \Psi_{\mathbf{k}, \alpha}^{\dagger}\left(A_{\mu, \mathbf{k}}\right)_{\alpha \beta} \Psi_{\mathbf{k}, \beta}=\frac{1}{V} \sum_{\mathbf{k}, \lambda \gamma} \Psi_{-\mathbf{k}, \lambda}^{\dagger}\left(\sigma_{1} A_{\mu, \mathbf{k}}^{T} \sigma_{1}\right)_{\lambda \gamma} \Psi_{-\mathbf{k}, \gamma}
$$

Hence, we gain the relation $\sigma_{1} A_{\mathbf{k}, \mu}^{T} \sigma_{1}=A_{\mu,-\mathbf{k}}$, which will be used repeatedly in the later proof. Second, the systematic linearresponse analysis needs a plain expression of the particle-hole space Green function, whose definition is $\mathcal{G}\left(\mathbf{k}, \tau ; \mathbf{k}^{\prime}, 0\right)_{i, j} \equiv$ $\mathcal{G}\left(\mathbf{k}, \mathbf{k}^{\prime} ; \tau\right)_{i, j} \equiv-\left\langle T_{\tau} \Psi_{\mathbf{k}, i}(\tau) \Psi_{\mathbf{k}^{\prime}, j}^{\dagger}(0)\right\rangle$. We derive the Green-function expression by virtue of its equation of motion,

$$
\partial_{\tau} \mathcal{G}\left(\mathbf{k}, \mathbf{k}^{\prime} ; \tau\right)_{\alpha \beta}=-\delta(\tau) \sigma_{3, \alpha \beta} \delta_{\mathbf{k}, \mathbf{k}^{\prime}}-\left(\sigma_{3} \mathcal{H}_{\mathbf{k}}\right)_{\alpha \gamma} \mathcal{G}\left(\mathbf{k}, \mathbf{k}^{\prime} ; \tau\right)_{\gamma \beta}
$$

where we used the relation

$$
\partial_{\tau} \Psi_{\mathbf{k}, \alpha}(\tau)=\left[H, \Psi_{\mathbf{k}, \alpha}(\tau)\right]=-\frac{1}{2}\left(\sigma_{3} \mathcal{H}_{\mathbf{k}}\right)_{\alpha \gamma} \Psi_{\mathbf{k}, \gamma}+\frac{i}{2} \Psi_{-\mathbf{k}, \gamma}^{\dagger}\left(\mathcal{H}_{-\mathbf{k}} \sigma_{2}\right)_{\gamma \alpha}=-\left(\sigma_{3} \mathcal{H}_{\mathbf{k}}\right)_{\alpha \gamma} \Psi_{\mathbf{k}, \gamma} .
$$

The equation of motion [Eq. (A6)] in matrix form reads

$$
\left(\partial_{\tau}+\sigma_{3} \mathcal{H}_{\mathbf{k}}\right) \mathcal{G}\left(\mathbf{k}, \mathbf{k}^{\prime} ; \tau\right)=-\sigma_{3} \delta(\tau) \delta_{\mathbf{k}, \mathbf{k}^{\prime}},
$$

so that $\mathcal{G}\left(\mathbf{k}, \mathbf{k}^{\prime} ; \tau\right)=\frac{-\sigma_{3} \delta(\tau) \delta_{\mathbf{k}, \mathbf{k}^{\prime}}}{\partial_{\tau}+\sigma_{3} \mathcal{H}_{\mathbf{k}}}$ and $\mathcal{G}\left(\mathbf{k}, \mathbf{k}^{\prime} ; i k_{n}\right)=\frac{\sigma_{3}}{i k_{n}-\sigma_{3} \mathcal{H}_{\mathbf{k}}} \delta_{\mathbf{k}, \mathbf{k}^{\prime}}$ in frequency-momentum space.

Now we show that the anomalous term in Eq. (A4) can be alternatively expressed, with the help of particle-hole symmetry, in the form of a Green function,

$$
\begin{aligned}
& \left\langle T_{\tau} \Psi_{\mathbf{k}, \alpha}^{\dagger}(\tau) \Psi_{\mathbf{k}, \rho}^{\dagger}(0)\right\rangle=\left\langle T_{\tau} \sigma_{1, \alpha \delta} \Psi_{-\mathbf{k}, \delta}(\tau) \Psi_{\mathbf{k}, \rho}^{\dagger}(0)\right\rangle=-\sigma_{1, \alpha \delta} \mathcal{G}\left(-\mathbf{k}, \mathbf{k}^{\prime} ; \tau\right)_{\delta \rho}, \\
& \left\langle T_{\tau} \Psi_{\mathbf{k}^{\prime}, \gamma}(\tau) \Psi_{\mathbf{k}, \sigma}(0)\right\rangle=\left\langle T_{\tau} \Psi_{\mathbf{k}, \gamma}(\tau) \Psi_{-\mathbf{k}^{\prime}, \mu}^{\dagger}(0) \sigma_{1, \mu \sigma}\right\rangle=-\mathcal{G}\left(\mathbf{k},-\mathbf{k}^{\prime} ; \tau\right)_{\gamma \mu} \sigma_{1, \mu \sigma} .
\end{aligned}
$$

Therefore, Eq. (A4) and the correlation tensor in Eq. (A3) are rewritten in terms of a Green function as

$$
\left\langle T_{\tau} \Psi_{\mathbf{k}, \alpha}^{\dagger}(\tau) \Psi_{\mathbf{k}, \gamma}(\tau) \Psi_{\mathbf{k}^{\prime}, \rho}^{\dagger}(0) \Psi_{\mathbf{k}^{\prime}, \sigma}(0)\right\rangle=\mathcal{G}_{\sigma \alpha}\left(\mathbf{k}^{\prime}, \mathbf{k} ;-\tau\right) \mathcal{G}_{\gamma \rho}\left(\mathbf{k}, \mathbf{k}^{\prime} ; \tau\right)+\left[\sigma_{1} \mathcal{G}\left(-\mathbf{k}, \mathbf{k}^{\prime} ; \tau\right)\right]_{\alpha \rho}\left[\mathcal{G}\left(\mathbf{k},-\mathbf{k}^{\prime} ; \tau\right) \sigma_{1}\right]_{\gamma \sigma},
$$


and

$$
\Pi_{\mu \nu}\left(i \omega_{m}\right)=-\frac{1}{V} \sum_{\mathbf{k}, \mathbf{k}^{\prime}} \int_{0}^{\beta} d \tau e^{i \omega_{m} \tau}\left(A_{\mu, \mathbf{k}}\right)_{\alpha \gamma}\left(J_{\nu, \mathbf{k}^{\prime}}^{q}\right)_{\rho \sigma}\left\{\mathcal{G}_{\sigma \alpha}\left(\mathbf{k}^{\prime}, \mathbf{k} ;-\tau\right) \mathcal{G}_{\gamma \rho}\left(\mathbf{k}, \mathbf{k}^{\prime} ; \tau\right)+\left[\sigma_{1} \mathcal{G}\left(-\mathbf{k}, \mathbf{k}^{\prime} ; \tau\right)\right]_{\alpha \rho}\left[\mathcal{G}\left(\mathbf{k},-\mathbf{k}^{\prime} ; \tau\right) \sigma_{1}\right]_{\gamma \sigma}\right\},
$$

respectively. Furthermore, with the aid of the Green-function relation $\mathcal{G}(-\mathbf{k}, \tau)=-\sigma_{1} \mathcal{G}(\mathbf{k},-\tau)^{T} \sigma_{1}$, we can prove the equivalence of the first and second part on the right-hand side of Eq. (A10). As a result, the correlation function becomes

$$
\Pi_{\mu \nu}\left(i \omega_{m}\right)=-\frac{2}{V} \sum_{\mathbf{k}} \int_{0}^{\beta} d \tau e^{i \omega_{m} \tau} \operatorname{tr}\left[A_{\mu, \mathbf{k}} \mathcal{G}(\mathbf{k}, \tau) J_{\nu, \mathbf{k}}^{q} \mathcal{G}(\mathbf{k} ;-\tau)\right],
$$

where $\mathcal{G}(\mathbf{k}, \tau)=\frac{\sigma_{3}}{i k_{n}-\sigma_{3} \mathcal{H}_{\mathbf{k}}}$. Let's transform the Green function to frequency space with $\mathcal{G}(\mathbf{k} ; \tau)=\frac{1}{\beta} \sum_{i q_{n}} e^{-i q_{n} \tau} \mathcal{G}\left(\mathbf{k} ; i q_{n}\right)$, then

$$
\Pi_{\mu \nu}\left(i \omega_{m}\right)=\frac{2}{V} \sum_{\mathbf{k}} \int_{-\infty}^{+\infty} \frac{d \omega_{1}}{2 \pi} \frac{d \omega_{2}}{2 \pi} \operatorname{tr}\left[A_{\mu, \mathbf{k}} S\left(\mathbf{k}, \omega_{1}\right) J_{\nu, \mathbf{k}}^{q} S\left(\mathbf{k}, \omega_{2}\right)\right] \frac{n_{\mathrm{B}}\left(\omega_{1}\right)-n_{\mathrm{B}}\left(\omega_{2}\right)}{\omega_{1}-\omega_{2}-i \omega_{m}} .
$$

Here, we performed the Matsubara summation and utilized $\mathcal{G}\left(\mathbf{k} ; i k_{n}\right)=\int_{-\infty}^{+\infty} \frac{d \omega}{2 \pi} \frac{S(\mathbf{k}, \omega)}{i k_{n}-\omega}$, with $S(\mathbf{k}, \omega)$ being the spectral function. Going back to the real time space and taking the zero frequency limit, we obtain the response tensor

$$
\begin{aligned}
K_{\mu \nu} & =-\left.i \frac{\partial \Pi_{\mu \nu}\left(\omega+i 0^{+}\right)}{\partial \omega}\right|_{\omega \rightarrow 0} \\
& =\frac{2}{V} \sum_{\mathbf{k}} \int_{-\infty}^{+\infty} \frac{d \varepsilon}{2 \pi} n_{\mathrm{B}}(\varepsilon) \operatorname{tr}\left[\left(G^{R}-G^{A}\right)\left(A_{\mu, \mathbf{k}} \frac{\partial G^{R}}{\partial \varepsilon} J_{\nu, \mathbf{k}}^{q}-J_{\nu, \mathbf{k}}^{q} \frac{\partial G^{A}}{\partial \varepsilon} A_{\mu, \mathbf{k}}\right)\right],
\end{aligned}
$$

where we used the relation

$$
\int_{-\infty}^{\infty} \frac{d \omega}{2 \pi} \frac{S(k, \omega)}{\left(\varepsilon-\omega \pm i 0^{+}\right)^{2}}=-\frac{\partial}{\partial \varepsilon} \int_{-\infty}^{\infty} \frac{d \omega}{2 \pi} \frac{S(k, \omega)}{\varepsilon-\omega \pm i 0^{+}}=-\frac{\partial G^{R / A}}{\partial \varepsilon}
$$

and the expression $S(\mathbf{k}, \varepsilon)=i\left(G^{R}-G^{A}\right)$.

\section{In the eigenstate basis}

To distinguish the intraband and interband contributions, we rewrite the response tensor in Eq. (A13) in the eigenstate basis via the transformation $\Psi_{\mathbf{k}}=T_{\mathbf{k}} \Gamma_{\mathbf{k}}$. By definition, we have the Green-function transformation $\mathcal{G}(\mathbf{k} ; \tau)=T_{\mathbf{k}} g(\mathbf{k}, \tau) T_{\mathbf{k}}^{\dagger}$, where $g(\mathbf{k}, \tau)=-\left\langle T_{\tau} \Gamma_{\mathbf{k}}(\tau) \Gamma_{\mathbf{k}}^{\dagger}(0)\right\rangle$ and $g^{R / A}(\mathbf{k}, \varepsilon)=\frac{\sigma_{3}}{\varepsilon-\sigma_{3} \mathcal{E}_{\mathbf{k}} \pm i 0^{+}}$. After this transformation, we obtain

$$
K_{\mu \nu}=\frac{2}{V} \sum_{\mathbf{k}} \int_{-\infty}^{+\infty} \frac{d \varepsilon}{2 \pi} n_{\mathrm{B}}(\varepsilon) \operatorname{tr}\left[\left(g^{R}-g^{A}\right)\left(\mathcal{A}_{\mu, \mathbf{k}} \frac{\partial g^{R}}{\partial \varepsilon} \mathcal{J}_{\nu, \mathbf{k}}-\mathcal{J}_{\nu, \mathbf{k}} \frac{\partial g^{A}}{\partial \varepsilon} \mathcal{A}_{\mu, \mathbf{k}}\right)\right],
$$

where $\mathcal{J}_{v, \mathbf{k}}=T_{\mathbf{k}}^{\dagger} J_{v, \mathbf{k}}^{q} T_{\mathbf{k}}$ and $\mathcal{A}_{\mu, \mathbf{k}}=T_{\mathbf{k}}^{\dagger} A_{\mu, \mathbf{k}} T_{\mathbf{k}}$. We split the expression into two parts: intraband and interband contributions. Owing to the hermitian conjugate property of operators, we write the response tensor elements as

$$
\begin{aligned}
K_{\mu \nu} & =\frac{2}{V} \sum_{\mathbf{k}} \sum_{m n} \int_{-\infty}^{+\infty} \frac{d \varepsilon}{2 \pi} n_{\mathrm{B}}(\varepsilon)\left[\left(g_{m}^{R}-g_{m}^{A}\right)\left(\left(\mathcal{A}_{\mu, \mathbf{k}}\right)_{m n} \frac{\partial g_{n}^{R}}{\partial \varepsilon}\left(\mathcal{J}_{\nu, \mathbf{k}}\right)_{n m}-\left(\mathcal{J}_{\nu, \mathbf{k}}\right)_{m n} \frac{\partial g_{n}^{A}}{\partial \varepsilon}\left(\mathcal{A}_{\mu, \mathbf{k}}\right)_{n m}\right)\right] \\
& =\frac{2 i}{V} \sum_{\mathbf{k}} \sum_{m n}\left(\mathcal{A}_{\mu, \mathbf{k}}\right)_{m n}\left(\mathcal{J}_{v, \mathbf{k}}\right)_{n m} \frac{\sigma_{3, m m} \sigma_{3, n n}\left\{n_{\mathrm{B}}\left[\left(\sigma_{3} \mathcal{E}_{\mathbf{k}}\right)_{m m}\right]-n_{\mathrm{B}}\left[\left(\sigma_{3} \mathcal{E}_{\mathbf{k}}\right)_{n n}\right]\right\}}{\left[\left(\sigma_{3} \mathcal{E}_{\mathbf{k}}\right)_{m m}-\left(\sigma_{3} \mathcal{E}_{\mathbf{k}}\right)_{n n}+i 0^{+}\right]^{2}},
\end{aligned}
$$

where we took the approximation $g_{m}^{R}-g_{m}^{A}=i 2 \operatorname{Im}\left(g_{m}^{R}\right)=-i 2 \pi \sigma_{3, m m} \delta\left[\varepsilon-\left(\sigma_{3} \mathcal{E}_{\mathbf{k}}\right)_{m m}\right]$. If we incorporate the magnon spectrum broadening $\Gamma_{m}$ into the Green function, i.e., $g_{m}^{R}(\varepsilon)=\frac{\sigma_{3, m m}}{\varepsilon-\left(\sigma_{3} \mathcal{E}_{\mathbf{k}}\right)_{m m}+i \Gamma_{m}}$, the response tensor can be naturally divided into two parts, $K_{\mu \nu}=K_{\mu \nu}^{\text {intra }}+K_{\mu \nu}^{\text {inter }}$, where

$$
K_{\mu \nu}^{\text {intra }}=\frac{1}{V} \sum_{\mathbf{k}} \sum_{n} \frac{1}{\Gamma_{n}}\left(\mathcal{J}_{\mathbf{k}, v}\right)_{n n}\left(\mathcal{A}_{\mu, \mathbf{k}}\right)_{n n} \partial_{\varepsilon} n_{\mathrm{B}}\left[\left(\sigma_{3} \mathcal{E}_{\mathbf{k}}\right)_{n n}\right]
$$

and

$$
K_{\mu \nu}^{\mathrm{inter}}=\frac{2 i}{V} \sum_{\mathbf{k}} \sum_{m \neq n}\left(\mathcal{A}_{\mu, \mathbf{k}}\right)_{m n}\left(\mathcal{J}_{\nu, \mathbf{k}}\right)_{n m} \frac{\sigma_{3, m m} \sigma_{3, n n}\left\{n_{\mathrm{B}}\left[\left(\sigma_{3} \mathcal{E}_{\mathbf{k}}\right)_{m m}\right]-n_{\mathrm{B}}\left[\left(\sigma_{3} \mathcal{E}_{\mathbf{k}}\right)_{n n}\right]\right\}}{\left[\left(\sigma_{3} \mathcal{E}_{\mathbf{k}}\right)_{m m}-\left(\sigma_{3} \mathcal{E}_{\mathbf{k}}\right)_{n n}\right]^{2}}
$$


The limit $\Gamma_{n} \rightarrow 0$ for $K_{\mu \nu}^{\text {inter }}$ is taken here. In consideration of $A_{\mu}^{\dagger}=A_{\mu}$ and $\left(J_{\nu}^{q}\right)^{\dagger}=J_{\nu}^{q}$, Eq. (A18) can be transformed to

$$
K_{\mu \nu}^{\text {inter }}=\frac{4}{V} \sum_{\mathbf{k}} \sum_{m \neq n} \frac{\operatorname{Im}\left[\left(\sigma_{3} \mathcal{A}_{\mu, \mathbf{k}}\right)_{n m}\left(\sigma_{3} \mathcal{J}_{\mathbf{k}, \nu}\right)_{m n}\right] n_{\mathrm{B}}\left[\left(\sigma_{3} \mathcal{E}_{\mathbf{k}}\right)_{n n}\right]}{\left[\left(\sigma_{3} \mathcal{E}_{\mathbf{k}}\right)_{m m}-\left(\sigma_{3} \mathcal{E}_{\mathbf{k}}\right)_{n n}\right]^{2}} .
$$

The intraband response Eq. (19) in the main text can be recovered if we consider $\mathcal{J}_{\mathbf{k}, v}=\frac{1}{4}\left(\mathcal{E}_{\mathbf{k}} \sigma_{3} \tilde{v}_{\mathbf{k}, v}+\tilde{v}_{\mathbf{k}, v} \sigma_{3} \mathcal{E}_{\mathbf{k}}\right)$ whose diagonal components read

$$
\left(\mathcal{J}_{\mathbf{k}, v}\right)_{n n}=\frac{1}{2}\left(\sigma_{3} \mathcal{E}_{\mathbf{k}}\right)_{n n}\left(\tilde{v}_{v, \mathbf{k}}\right)_{n n},
$$

where

$$
\tilde{v}_{\mathbf{k}, v}=\partial_{k_{v}} \mathcal{E}_{\mathbf{k}}-\left(\partial_{k_{v}} T_{\mathbf{k}}^{\dagger}\right) \mathcal{H}_{\mathbf{k}} T_{\mathbf{k}}-T_{\mathbf{k}}^{\dagger} \mathcal{H}_{\mathbf{k}}\left(\partial_{k_{v}} T_{\mathbf{k}}\right)
$$

From the paraunitary relation of $T_{\mathbf{k}}$ and $\partial_{k_{v}}\left(T_{\mathbf{k}} \sigma_{3} T_{\mathbf{k}}^{\dagger}\right)=0$, we get $\partial_{k_{v}} T_{\mathbf{k}}^{\dagger}=-T_{\mathbf{k}} \sigma_{3}\left(\partial_{k_{v}} T_{\mathbf{k}}\right) \sigma_{3} T_{\mathbf{k}}^{\dagger}$. From $T_{\mathbf{k}}^{\dagger} \mathcal{H}_{\mathbf{k}} T_{\mathbf{k}}=\mathcal{E}_{\mathbf{k}}$ and $\left(T_{\mathbf{k}}\right)^{-1}=$ $\sigma_{3} T_{\mathbf{k}}^{\dagger} \sigma_{3}$, we have $T_{\mathbf{k}}^{\dagger} \mathcal{H}_{\mathbf{k}}=\mathcal{E}_{\mathbf{k}} \sigma_{3} T_{\mathbf{k}}^{\dagger} \sigma_{3}$. Therefore, the diagonal elements of $\tilde{v}_{\mathbf{k}, v}$ are shown to be

$$
\left(\tilde{v}_{\mathbf{k}, v}\right)_{n n}=\left(\partial_{k_{v}} \mathcal{E}_{\mathbf{k}}\right)_{n n}+\left(T_{\mathbf{k}}^{\dagger} \sigma_{3} \partial_{k_{v}} T_{\mathbf{k}} \sigma_{3} \mathcal{E}_{\mathbf{k}}\right)_{n n}-\left(\mathcal{E}_{\mathbf{k}} \sigma_{3} T_{\mathbf{k}}^{\dagger} \sigma_{3} \partial_{k_{\nu}} T_{\mathbf{k}}\right)_{n n}=\left(\partial_{\nu} \mathcal{E}_{\mathbf{k}}\right)_{n n} ;
$$

thus,

$$
\left(\mathcal{J}_{\mathbf{k}, v}\right)_{n n}=\frac{1}{2}\left(\sigma_{3} \mathcal{E}_{\mathbf{k}}\right)_{n n}\left(\partial_{k_{v}} \mathcal{E}_{\mathbf{k}}\right)_{n n} .
$$

By inserting Eq. (A23) into Eq. (A17), we arrive at

$$
K_{\mu \nu}^{\mathrm{intra}}=\frac{1}{V} \sum_{\mathbf{k}} \sum_{n=1}^{2 N} \frac{1}{2 \Gamma_{n}}\left(\mathcal{A}_{\mu, \mathbf{k}}\right)_{n n} \partial_{k_{\nu}} \mathcal{E}_{\mathbf{k}, n n}\left(\sigma_{3} \mathcal{E}_{\mathbf{k}}\right)_{n n} \partial_{\varepsilon} n_{\mathrm{B}}\left[\left(\sigma_{3} \mathcal{E}_{\mathbf{k}}\right)_{n n}\right] .
$$

Given the relation $n_{\mathrm{B}}(x)=-1-n_{\mathrm{B}}(-x)$, the band index can be confined to the particle space, i.e., $1 \leqslant n \leqslant N$,

$$
K_{\mu \nu}^{\text {intra }}=\frac{1}{V} \sum_{\mathbf{k}} \sum_{n=1}^{N} \frac{1}{2 \Gamma_{n}}\left[\left(\mathcal{A}_{\mu, \mathbf{k}}\right)_{n n}+\left(\mathcal{A}_{\mu,-\mathbf{k}}\right)_{(n+N)(n+N)}\right] \partial_{k_{\nu}} \mathcal{E}_{\mathbf{k}, n n} \mathcal{E}_{\mathbf{k}, n n} \partial_{\varepsilon} n_{\mathrm{B}}\left[\mathcal{E}_{\mathbf{k}, n n}\right] .
$$

Applying particle-hole symmetry (PHS), $\left(\mathcal{A}_{\mu, \mathbf{k}}\right)_{n n}=\left(\mathcal{A}_{\mu,-\mathbf{k}}\right)_{(n+N)(n+N)}$, replacing $\mathcal{A}_{\mu, \mathbf{k}}$ by $\mathcal{S}_{\mu, \mathbf{k}}$ and taking $\nabla_{\nu} \phi=-\nabla_{\nu} T / T$ into account, we can obtain the intraband response Eq. (19).

On the other hand, by plugging the expression of $\mathcal{J}_{\nu, \mathbf{k}}$ into Eqs. (A18) or (A19), the interband part can be reorganized as below:

$$
\begin{aligned}
K_{\mu \nu}^{\text {inter }} & =\frac{1}{V} \sum_{\mathbf{k}} \sum_{m \neq n} \frac{i}{2}\left(\mathcal{A}_{\mu, \mathbf{k}}\right)_{n m}\left[\left(\sigma_{3} \mathcal{E}_{\mathbf{k}}\right)_{m m}\left(v_{\nu}\right)_{m n}+\left(v_{\nu}\right)_{m n}\left(\sigma_{3} \mathcal{E}_{\mathbf{k}}\right)_{n n}\right] \frac{\sigma_{3, m m} \sigma_{3, n n}\left\{n_{\mathrm{B}}\left[\left(\sigma_{3} \mathcal{E}_{\mathbf{k}}\right)_{n n}\right]-n_{\mathrm{B}}\left[\left(\sigma_{3} \mathcal{E}_{\mathbf{k}}\right)_{m m}\right]\right\}}{\left[\left(\sigma_{3} \mathcal{E}_{\mathbf{k}}\right)_{m m}-\left(\sigma_{3} \mathcal{E}_{\mathbf{k}}\right)_{n n}\right]^{2}} \\
& =\frac{1}{V} \sum_{\mathbf{k}} \sum_{n=1}^{2 N}-\left(\Omega_{n, \mathbf{k}}^{A}\right)_{\mu \nu} \bar{\varepsilon}_{n, \mathbf{k}} n_{\mathrm{B}}\left(\bar{\varepsilon}_{n, \mathbf{k}}\right)-\left(m_{n, \mathbf{k}}^{A}\right)_{\mu \nu} n_{\mathrm{B}}\left(\bar{\varepsilon}_{n, \mathbf{k}}\right),
\end{aligned}
$$

with

$$
\left(\Omega_{n, \mathbf{k}}^{A}\right)_{\mu \nu}=\sum_{m(\neq n)} \frac{2 \operatorname{Im}\left[\left(\sigma_{3} \mathcal{A}_{\mu, \mathbf{k}}\right)_{n m}\left(\sigma_{3} \tilde{v}_{v, \mathbf{k}}\right)_{m n}\right]}{\left(\bar{\varepsilon}_{n, \mathbf{k}}-\bar{\varepsilon}_{m, \mathbf{k}}\right)^{2}}, \quad\left(m_{n, \mathbf{k}}^{A}\right)_{\mu \nu}=\sum_{m(\neq n)} \frac{-\operatorname{Im}\left[\left(\sigma_{3} \mathcal{A}_{\mu, \mathbf{k}}\right)_{n m}\left(\sigma_{3} \tilde{v}_{v, \mathbf{k}}\right)_{m n}\right]}{\bar{\varepsilon}_{n, \mathbf{k}}-\bar{\varepsilon}_{m, \mathbf{k}}} .
$$

When we identify the operator $\hat{A}_{\mu}$ as magnon spin operator $\hat{\Sigma}_{\mu}$, the tensors above become spin-dependent tensors,

$$
\begin{aligned}
\left(\Omega_{n, \mathbf{k}}^{\Sigma}\right)_{\mu \nu} & =\sum_{m(\neq n)}\left(\sigma_{3}\right)_{n n}\left(\sigma_{3}\right)_{m m} \frac{2 \operatorname{Im}\left(\left\langle u_{n, \mathbf{k}}\left|\Sigma_{\mu}\right| u_{m, \mathbf{k}}\right\rangle\left\langle u_{m, \mathbf{k}}\left|v_{\nu}\right| u_{n, \mathbf{k}}\right\rangle\right)}{\left(\bar{\varepsilon}_{n, \mathbf{k}}-\bar{\varepsilon}_{m, \mathbf{k}}\right)^{2}}, \\
\left(m_{n, \mathbf{k}}^{\Sigma}\right)_{\mu \nu} & =-\sum_{m(\neq n)}\left(\sigma_{3}\right)_{n n}\left(\sigma_{3}\right)_{m m} \frac{\operatorname{Im}\left(\left\langle u_{n, \mathbf{k}}\left|\Sigma_{\mu}\right| u_{m, \mathbf{k}}\right\rangle\left\langle u_{m, \mathbf{k}}\left|v_{\nu}\right| u_{n, \mathbf{k}}\right\rangle\right)}{\bar{\varepsilon}_{n, \mathbf{k}}-\bar{\varepsilon}_{m, \mathbf{k}}} .
\end{aligned}
$$

The tensor $\left(\Omega_{n, \mathbf{k}}^{\Sigma}\right)_{\mu \nu}$ defined in Eq. (A28) exists in both particle and hole space, and we can verify that this tensor fulfills two relations:

(1) Summation rule:

$$
\sum_{n=1}^{2 N}\left(\Omega_{n, \mathbf{k}}^{\Sigma}\right)_{\mu \nu}=\sum_{m \neq n}\left(\sigma_{3}\right)_{n n}\left(\sigma_{3}\right)_{m m} \operatorname{Im}\left[\frac{\left\langle u_{n, \mathbf{k}}\left|\Sigma_{\mu}\right| u_{m, \mathbf{k}}\right\rangle\left\langle u_{m, \mathbf{k}}\left|v_{\nu}\right| u_{n, \mathbf{k}}\right\rangle}{\left(\bar{\varepsilon}_{n, \mathbf{k}}-\bar{\varepsilon}_{m, \mathbf{k}}\right)^{2}}+\frac{\left\langle u_{m, \mathbf{k}}\left|\Sigma_{\mu}\right| u_{n, \mathbf{k}}\right\rangle\left\langle u_{n, \mathbf{k}}\left|v_{\mu}\right| u_{m, \mathbf{k}}\right\rangle}{\left(\bar{\varepsilon}_{n, \mathbf{k}}-\bar{\varepsilon}_{m, \mathbf{k}}\right)^{2}}\right]=0 .
$$

In the middle step, we utilized the property that the band indices $m, n$ can be interchanged. 
(2) Mapping between particle and hole space. We note that the velocity $v_{\mathbf{k}}$ satisfies

$$
\sigma_{1} v_{\mathbf{k}} \sigma_{1}=\sigma_{1} \frac{\partial H_{\mathbf{k}}}{\partial \mathbf{k}} \sigma_{1}=-v_{-\mathbf{k}}^{*} .
$$

At the same time, the spin operator obey particle-hole symmetry,

$$
\sigma_{1} \Sigma_{\mu} \sigma_{1}=\Sigma_{\mu}
$$

Using the particle-hole symmetry property of the eigenstates and eigenvalues, we are able to show

$$
\begin{aligned}
\left(\Omega_{n, \mathbf{k}}^{\Sigma}\right)_{\mu \nu} & =\sum_{m(\neq n)}\left(\sigma_{3}\right)_{n n}\left(\sigma_{3}\right)_{m m} \frac{2 \operatorname{Im}\left(\left\langle u_{n, \mathbf{k}}\left|\Sigma_{\mu}\right| u_{m, \mathbf{k}}\right\rangle\left\langle u_{m, \mathbf{k}}\left|v_{v, \mathbf{k}}\right| u_{n, \mathbf{k}}\right\rangle\right)}{\left(\bar{\varepsilon}_{n, \mathbf{k}}-\bar{\varepsilon}_{m, \mathbf{k}}\right)^{2}} \\
& =\sum_{m+N(\neq n+N)}\left(\sigma_{3}\right)_{n+N, n+N}\left(\sigma_{3}\right)_{m+N, m+N} \frac{2 \operatorname{Im}\left(\left\langle u_{n+N,-\mathbf{k}}^{*}\left|\sigma_{1} \Sigma_{\mu} \sigma_{1}\right| u_{m+N,-\mathbf{k}}^{*}\right\rangle\left\langle u_{m+N,-\mathbf{k}}^{*}\left|\sigma_{1} v_{\nu, \mathbf{k}} \sigma_{1}\right| u_{n+N,-\mathbf{k}}^{*}\right\rangle\right)}{\left(\bar{\varepsilon}_{n+N,-\mathbf{k}}-\bar{\varepsilon}_{m+N,-\mathbf{k}}\right)^{2}} \\
& =\sum_{m(\neq n+N)}\left(\sigma_{3}\right)_{n+N, n+N}\left(\sigma_{3}\right)_{m m} \frac{2 \operatorname{Im}\left[\left(\left\langle u_{n+N,-\mathbf{k}}\left|\Sigma_{\mu}\right| u_{m,-\mathbf{k}}\right\rangle\right)^{*}\left(\left\langle u_{m,-\mathbf{k}}\left|-v_{\nu,-\mathbf{k}}\right| u_{n+N,-\mathbf{k}}\right\rangle\right)^{*}\right]}{\left(\bar{\varepsilon}_{n+N,-\mathbf{k}}-\bar{\varepsilon}_{m,-\mathbf{k}}\right)^{2}} \\
& =\left(\Omega_{n+N,-\mathbf{k}}^{\Sigma}\right)_{\mu \nu} .
\end{aligned}
$$

\section{Detailed calculation of dipole contribution coefficient $D_{\mu \nu}$}

As an example, we calculate $D_{y x}$ by choosing $\mathbf{B}=\frac{B}{q} \sin \left(\mathbf{q}_{1} \cdot \mathbf{r}\right) \hat{\mathbf{e}}_{y}$ with $\mathbf{q}_{1}=q \hat{\mathbf{e}}_{x}$. Substituting Eq. (31) into Eq. (30), we obtain

$$
\begin{aligned}
\tilde{D}_{y x} & =\lim _{q \rightarrow 0} \frac{1}{2 V} \sum_{\mathbf{k}} \sum_{m n} \frac{1}{i 2 q} g\left(\bar{\varepsilon}_{n \mathbf{k}}\right)\left(\sigma_{3}\right)_{n n}\left(\sigma_{3}\right)_{m m} \bar{\varepsilon}_{n \mathbf{k}}\left[\frac{\left\langle u_{n \mathbf{k}}\left|\sigma_{3}\right| u_{m, \mathbf{k}+\mathbf{q}_{1}}\right\rangle\left\langle u_{m, \mathbf{k}+\mathbf{q}_{1}}\left|\left(\Sigma_{y, \mathbf{k}}+\Sigma_{y, \mathbf{k}+\mathbf{q}_{1}}\right)\right| u_{n, \mathbf{k}}\right\rangle}{\bar{\varepsilon}_{n \mathbf{k}}-\bar{\varepsilon}_{m, \mathbf{k}+\mathbf{q}_{1}}}-\left(\mathbf{q}_{1} \rightarrow-\mathbf{q}_{1}\right)\right]+\text { c.c. } \\
& =\lim _{q \rightarrow 0} \frac{1}{2 V} \sum_{\mathbf{k}} \sum_{m n} \frac{1}{i 2 q}\left[g\left(\bar{\varepsilon}_{n \mathbf{k}}\right) \bar{\varepsilon}_{n \mathbf{k}}-g\left(\bar{\varepsilon}_{m, \mathbf{k}+\mathbf{q}_{1}}\right) \bar{\varepsilon}_{m, \mathbf{k}+\mathbf{q}_{1}}\right]\left(\sigma_{3}\right)_{n n}\left(\sigma_{3}\right)_{m m} \frac{\left\langle u_{n \mathbf{k}}\left|\sigma_{3}\right| u_{m, \mathbf{k}+\mathbf{q}_{1}}\right\rangle\left\langle u_{m, \mathbf{k}+\mathbf{q}_{1}}\left|\left(\Sigma_{y, \mathbf{k}}+\Sigma_{y, \mathbf{k}+\mathbf{q}_{1}}\right)\right| u_{n, \mathbf{k}}\right\rangle}{\bar{\varepsilon}_{n \mathbf{k}}-\bar{\varepsilon}_{m, \mathbf{k}+\mathbf{q}_{1}}}+\text { c.c. }
\end{aligned}
$$

Taking the limit, we get for $m \neq n$,

$$
\begin{aligned}
\left(\tilde{D}_{y x}\right)_{1} & =\frac{1}{V} \sum_{\mathbf{k}} \sum_{m \neq n} \frac{1}{2}\left[g\left(\bar{\varepsilon}_{m \mathbf{k}}\right) \bar{\varepsilon}_{m \mathbf{k}}-g\left(\bar{\varepsilon}_{n, \mathbf{k}}\right) \bar{\varepsilon}_{n, \mathbf{k}}\right]\left(\sigma_{3}\right)_{n n}\left(\sigma_{3}\right)_{m m} \frac{i\left\langle u_{n, \mathbf{k}}\left|\sigma_{3}\right| \partial_{k_{x}} u_{m, \mathbf{k}}\right\rangle\left\langle u_{m, \mathbf{k}}\left|\Sigma_{y}\right| u_{n, \mathbf{k}}\right\rangle}{\bar{\varepsilon}_{n, \mathbf{k}}-\bar{\varepsilon}_{m, \mathbf{k}}}+\text { c.c. } \\
& =\frac{1}{V} \sum_{\mathbf{k}} \sum_{m \neq n}-\frac{1}{2}\left[g\left(\bar{\varepsilon}_{m \mathbf{k}}\right) \bar{\varepsilon}_{m \mathbf{k}}-g\left(\bar{\varepsilon}_{n, \mathbf{k}}\right) \bar{\varepsilon}_{n, \mathbf{k}}\right]\left(\sigma_{3}\right)_{m n}\left(\sigma_{3}\right)_{m m} \frac{i\left\langle u_{n, \mathbf{k}}\left|v_{x}\right| u_{m, \mathbf{k}}\right\rangle\left\langle u_{m, \mathbf{k}}\left|\Sigma_{y}\right| u_{n, \mathbf{k}}\right\rangle}{\left(\bar{\varepsilon}_{n, \mathbf{k}}-\bar{\varepsilon}_{m, \mathbf{k}}\right)^{2}}+\text { c.c.. }
\end{aligned}
$$

For $m=n$, we have

$$
\begin{aligned}
\left(\tilde{D}_{y x}\right)_{2} & =\frac{1}{V} \sum_{\mathbf{k}} \sum_{n} \frac{1}{2 i}\left[g\left(\bar{\varepsilon}_{n, \mathbf{k}}\right)+g^{\prime}\left(\bar{\varepsilon}_{n, \mathbf{k}}\right) \bar{\varepsilon}_{n, \mathbf{k}}\right]\left[\left\langle u_{n, \mathbf{k}} \mid \sigma_{3} \partial_{k_{x}} u_{n, \mathbf{k}}\right\rangle\left\langle u_{n, \mathbf{k}}\left|\Sigma_{y}\right| u_{n, \mathbf{k}}\right\rangle+\left(\sigma_{3}\right)_{n n}\left\langle\partial_{k_{x}} u_{n, \mathbf{k}}\left|\Sigma_{y}\right| u_{n, \mathbf{k}}\right\rangle\right]+\text { c.c. } \\
& =\frac{1}{V} \sum_{\mathbf{k}} \sum_{n}-\frac{1}{2}\left[g\left(\bar{\varepsilon}_{n, \mathbf{k}}\right)+g^{\prime}\left(\bar{\varepsilon}_{n, \mathbf{k}}\right) \bar{\varepsilon}_{n, \mathbf{k}}\right]\left(\sigma_{3}\right)_{n n}\left(\sigma_{3}\right)_{m m} \frac{i\left\langle u_{n, \mathbf{k}}\left|v_{x}\right| u_{m, \mathbf{k}}\right\rangle\left\langle u_{m, \mathbf{k}}\left|\Sigma_{y}\right| u_{n, \mathbf{k}}\right\rangle}{\bar{\varepsilon}_{n, \mathbf{k}}-\bar{\varepsilon}_{m, \mathbf{k}}}+\text { c.c. }
\end{aligned}
$$

Above $v_{x}=\partial_{k_{x}} H$. In total, we have

$$
\tilde{D}_{y x}=\left(\tilde{D}_{y x}\right)_{1}+\left(\tilde{D}_{y x}\right)_{2}=\frac{1}{V} \sum_{n \mathbf{k}} g\left(\bar{\varepsilon}_{n \mathbf{k}}\right) \bar{\varepsilon}_{n \mathbf{k}}\left(\Omega_{n, \mathbf{k}}^{\Sigma}\right)_{y x}+\left[g\left(\bar{\varepsilon}_{n, \mathbf{k}}\right)+g^{\prime}\left(\bar{\varepsilon}_{n, \mathbf{k}}\right) \bar{\varepsilon}_{n, \mathbf{k}}\right]\left(m_{n, \mathbf{k}}^{\Sigma}\right)_{y x}
$$

The calculation of the other components is analogous and the general result is

$$
\tilde{D}_{\mu \nu}=\frac{1}{V} \sum_{n \mathbf{k}} g\left(\bar{\varepsilon}_{n \mathbf{k}}\right) \bar{\varepsilon}_{n \mathbf{k}}\left(\Omega_{n, \mathbf{k}}^{\Sigma}\right)_{\mu \nu}+\left[g\left(\bar{\varepsilon}_{n, \mathbf{k}}\right)+g^{\prime}\left(\bar{\varepsilon}_{n, \mathbf{k}}\right) \bar{\varepsilon}_{n, \mathbf{k}}\right]\left(m_{n, \mathbf{k}}^{\Sigma}\right)_{\mu \nu} .
$$

Finally, by using Eq. (28) we obtain

$$
D_{\mu \nu}=\frac{1}{\beta} \int_{0}^{\beta} d \bar{\beta} \tilde{D}_{\mu \nu}=\frac{1}{V} \sum_{n \mathbf{k}}\left(\left(\Omega_{n, \mathbf{k}}^{\Sigma}\right)_{\mu \nu} \int_{0}^{\bar{\varepsilon}_{n \mathbf{k}}} d \eta g(\eta)+\left(m_{n, \mathbf{k}}^{\Sigma}\right)_{\mu \nu} g\left(\bar{\varepsilon}_{n, \mathbf{k}}\right)\right) .
$$

Here we used the relation $\frac{1}{\beta} \int_{0}^{\beta} d \bar{\beta} g\left(\bar{\varepsilon}_{n, \mathbf{k}}\right) \bar{\varepsilon}_{n, \mathbf{k}}=\int_{0}^{\bar{\varepsilon}_{n \mathbf{k}}} d \eta g(\eta)$ with $g(\eta)=\frac{1}{e^{\beta \eta}-1}$ and $\frac{d}{d \bar{\beta}}\left[\bar{\beta} g\left(\bar{\varepsilon}_{n, \mathbf{k}}\right)\right]=g\left(\bar{\varepsilon}_{n, \mathbf{k}}\right)+g^{\prime}\left(\bar{\varepsilon}_{n, \mathbf{k}}\right) \bar{\varepsilon}_{n, \mathbf{k}}$. 


\section{Total intrinsic response coefficient $\chi_{\mu \nu}^{\text {in }}$}

Adding the Kubo formula Eq. (22) and dipole moment contributions Eq. (34) [or Eq. (22)], the total response reads

$$
\begin{aligned}
K_{\mu \nu}^{\mathrm{inter}}+D_{\mu \nu} & =\frac{1}{V} \sum_{n \mathbf{k}}\left(\Omega_{n, \mathbf{k}}^{\Sigma}\right)_{\mu \nu}\left[-\bar{\varepsilon}_{n \mathbf{k}} g\left(\bar{\varepsilon}_{n \mathbf{k}}\right)+\int_{0}^{\bar{\varepsilon}_{n \mathbf{k}}} d \eta g(\eta)\right]=-\frac{1}{V} \sum_{n \mathbf{k}}\left(\Omega_{n, \mathbf{k}}^{\Sigma}\right)_{\mu \nu} \int_{0}^{\bar{\varepsilon}_{n \mathbf{k}}} d \eta \eta \frac{d g(\eta)}{d \eta} \\
& =-\frac{1}{V} \sum_{n \mathbf{k}}\left(\Omega_{n, \mathbf{k}}^{\Sigma}\right)_{\mu \nu} \tilde{c}_{1}\left(\bar{\varepsilon}_{n \mathbf{k}}\right),
\end{aligned}
$$

where $\tilde{c}_{1}(x)=\int_{0}^{x} d \eta \eta \frac{d g(\eta)}{d \eta}$ with $g(\eta)=\frac{1}{e^{\beta \eta}-1}$. Using the relation $-g(-\eta)=1+g(\eta)$, we have $\tilde{c}(x)=\tilde{c}(-x)$. Therefore, the response function can be reduced to

$$
\begin{aligned}
K_{\mu \nu}^{\text {inter }}+D_{\mu \nu} & =-\frac{1}{V} \sum_{n=1}^{N} \sum_{\mathbf{k}}\left[\left(\Omega_{n, \mathbf{k}}^{\Sigma}\right)_{\mu \nu} \tilde{c}_{1}\left(\varepsilon_{n \mathbf{k}}\right)+\left(\Omega_{n+N, \mathbf{k}}^{\Sigma}\right)_{\mu \nu} \tilde{c}_{1}\left(-\varepsilon_{n,-\mathbf{k}}\right)\right]=-\frac{1}{V} \sum_{n=1}^{N} \sum_{\mathbf{k}}\left[\left(\Omega_{n, \mathbf{k}}^{\Sigma}\right)_{\mu \nu}+\left(\Omega_{n+N,-\mathbf{k}}^{\Sigma}\right)_{\mu \nu}\right] \tilde{c}_{1}\left(\varepsilon_{n, \mathbf{k}}\right) \\
& =-\frac{1}{V} \sum_{n=1}^{N} \sum_{\mathbf{k}}\left[\left(\Omega_{n, \mathbf{k}}^{\Sigma}\right)_{\mu \nu}+\left(\Omega_{n+N,-\mathbf{k}}^{\Sigma}\right)_{\mu \nu}\right]\left(\tilde{c}_{1}\left(\varepsilon_{n, \mathbf{k}}\right)-\int_{0}^{\infty} d \eta \eta \frac{d g(\eta)}{d \eta}\right) \\
& =-\frac{k_{B} T}{V} \sum_{n=1}^{N} \sum_{\mathbf{k}}\left[\left(\Omega_{n, \mathbf{k}}^{\Sigma}\right)_{\mu \nu}+\left(\Omega_{n+N,-\mathbf{k}}^{\Sigma}\right)_{\mu \nu}\right] c_{1}\left[g\left(\varepsilon_{n, \mathbf{k}}\right)\right]=-\frac{2 k_{B} T}{V} \sum_{n=1}^{N} \sum_{\mathbf{k}}\left(\Omega_{n, \mathbf{k}}^{\Sigma}\right)_{\mu \nu} c_{1}\left[g\left(\varepsilon_{n, \mathbf{k}}\right)\right]
\end{aligned}
$$

Here we used the properties of Berry curvature shown in Eqs. (A30) and (A33), and the relation $-\int_{\varepsilon_{n}}^{\infty} \eta \frac{d g(\eta)}{d \eta}=\frac{1}{\beta} c_{1}\left[g\left(\varepsilon_{n}\right)\right]$. Considering the relation $\chi_{\mu \nu}^{\text {in }} \nabla_{\nu} T=\left(K_{\mu \nu}^{\text {inter }}+D_{\mu \nu}\right) \nabla_{\nu} \phi$ and $\nabla_{\nu} \phi=-\nabla_{\nu} T / T$, we obtain the total intrinsic response coefficient in Eq. (35).

\section{APPENDIX B: DETAILS OF THE MODELS}

\section{Antiferromagnetic spin chain}

We recapitulate that the Hamiltonian for the antiferromagnetic spin chain is

$$
H=\sum_{i} \sum_{\delta= \pm 1}\left[J\left(\gamma S_{1, i}^{x} S_{2, i+\nu}^{x}+S_{1, i}^{y} S_{2, i+\nu}^{y}+\lambda S_{1, i}^{z} S_{2, i+\nu}^{z}\right)+D_{12}^{v} \mathbf{e}_{z} \cdot\left(\mathbf{S}_{1, i} \times \mathbf{S}_{2, i+\nu}\right)\right]
$$

with exchange and DMI parameters as stated in the main text. After performing the Holstein-Primakoff transformation, the quadratic Hamiltonian written in the basis $\Psi_{k}=\left(a_{1, k}, a_{2, k}, a_{1,-k}^{\dagger}, a_{2,-k}^{\dagger}\right)^{T}$ reads

$$
\mathcal{H}_{k}=J S\left[\begin{array}{cccc}
2 \lambda & 2 \Delta_{-} \cos k & 0 & 2 \Delta_{+} \cos k+i \varrho_{k} \\
2 \Delta_{-} \cos k & 2 \lambda & 2 \Delta_{+} \cos k+i \varrho_{-k} & 0 \\
0 & 2 \Delta_{+} \cos k-i \varrho_{k} & 2 \lambda & 2 \Delta_{-} \cos k \\
2 \Delta_{+} \cos k-i \varrho_{-k} & 0 & 2 \Delta_{-} \cos k & 2 \lambda
\end{array}\right]
$$

where $\Delta_{ \pm}=\frac{1 \pm \gamma}{2}, \varrho_{k}=\sum_{v} \delta D_{v} e^{i k v} / J=i 2 D_{0} \sin k+2 \delta_{D} \cos k$, with $D_{0}=\frac{D_{1}+D_{2}}{2 J}$ and $\delta_{D}=\frac{D_{1}-D_{2}}{2 J}$.

\section{Noncoplanar kagome antiferromagnet}

We consider the noncoplanar kagome antiferromagnet described by

$$
H=\sum_{\langle i j\rangle} J_{1} \mathbf{S}_{i} \cdot \mathbf{S}_{j}+\mathbf{D}_{i j} \cdot\left(\mathbf{S}_{i} \times \mathbf{S}_{j}\right)+\sum_{\langle\langle i j\rangle\rangle} J_{2} \mathbf{S}_{i} \cdot \mathbf{S}_{j},
$$

where $\mathbf{D}_{i j}=\mathbf{D}_{p, i j}+D_{z, i j} \hat{z}$. The spins cant out of the 2D plane with a small angle $\eta$, and the spins' projection on the the $x-y$ plane forms angles $\theta_{i}(i=1,2,3)$ with respect to the $x$ axis, specifically, $\theta_{1}=-\pi / 6, \theta_{2}=\pi / 2$, and $\theta_{3}=7 \pi / 6$. For each spin $\mathbf{S}_{i}$, we choose a local reference frame defined as follows:

$$
\mathbf{e}_{i, x}=\left\{\sin \theta_{i}-\cos \theta_{i}, 0\right\}, \quad \mathbf{e}_{i, y}=\left\{\sin \eta \cos \theta_{i}, \sin \eta \sin \theta_{i},-\cos \eta\right\}, \quad \mathbf{e}_{i, z}=\left\{\cos \eta \cos \theta_{i}, \cos \eta \sin \theta_{i}, \sin \eta\right\} .
$$


For a given spin $\mathbf{S}_{i}$, in the global frame, its components can be connected to the local frame expression $\tilde{\mathbf{S}}_{i}$ by

$$
S_{i}^{\alpha}=\mathbf{e}_{\alpha} \cdot\left(\tilde{S}_{i}^{\beta} \mathbf{e}_{i, \beta}\right)=R_{i, \alpha \beta} \tilde{S}_{i}^{\beta},
$$

where $R_{i, \alpha \beta}=\mathbf{e}_{\alpha} \cdot \mathbf{e}_{i, \beta}$, or in matrix form,

$$
R_{i}=\left(\begin{array}{ccc}
\sin \theta_{i} & \sin \eta \cos \theta_{i} & \cos \eta \cos \theta_{i} \\
-\cos \theta_{i} & \sin \eta \sin \theta_{i} & \cos \eta \sin \theta_{i} \\
0 & -\cos \eta & \sin \eta
\end{array}\right)
$$

For the general spin-spin interaction a correspondence between the two frames can be written as $S_{i}^{\alpha} \Gamma_{\alpha \beta}^{i j} S_{j}^{\beta}=\tilde{S}_{i}^{\alpha}\left(R_{i}^{T} \Gamma^{i j} R_{j}\right)_{\alpha \beta} \tilde{S}_{j}^{\beta}$. The interaction matrices are $\Gamma_{\alpha \beta}^{i j}=J \delta_{\alpha \beta}$ for exchange and $\Gamma_{\alpha \beta}^{i j}=D_{i j}^{\rho} \epsilon^{\rho \alpha \beta}$ for DMI. Using these relations, we express the noninteracting magnon Hamiltonian in terms of the local reference frames as

$$
\begin{aligned}
& H_{J_{1}}=J_{1} \sum_{\langle i j\rangle} \cos \theta_{i j} \tilde{\mathbf{S}}_{i} \cdot \tilde{\mathbf{S}}_{j}+2 \sin ^{2}\left(\theta_{i j} / 2\right)\left(\cos ^{2} \eta \tilde{S}_{i}^{y} \tilde{S}_{j}^{y}+\sin ^{2} \eta \tilde{S}_{i}^{z} \tilde{S}_{j}^{z}\right)+\sin \eta \sin \theta_{i j} \hat{z} \cdot\left(\tilde{\mathbf{S}}_{i} \times \tilde{\mathbf{S}}_{j}\right), \\
& H_{J_{2}}=J_{2} \sum_{\langle\langle i j\rangle\rangle} \cos \theta_{i j} \tilde{\mathbf{S}}_{i} \cdot \tilde{\mathbf{S}}_{j}+2 \sin ^{2}\left(\theta_{i j} / 2\right)\left(\cos ^{2} \eta \tilde{S}_{i}^{y} \tilde{S}_{j}^{y}+\sin ^{2} \eta \tilde{S}_{i}^{z} \tilde{S}_{j}^{z}\right)+\sin \eta \sin \theta_{i j} \hat{z} \cdot\left(\tilde{\mathbf{S}}_{i} \times \tilde{\mathbf{S}}_{j}\right), \\
& H_{D_{z}}=\sum_{\langle i j\rangle}-s_{i j} D_{z}\left[\sin \theta_{i j}\left(\tilde{S}_{i}^{x} \tilde{S}_{j}^{x}+\sin ^{2} \eta \tilde{S}_{i}^{y} \tilde{S}_{j}^{y}+\cos ^{2} \eta \tilde{S}_{i}^{z} \tilde{S}_{j}^{z}\right)-\sin \eta \cos \theta_{i j} \hat{z} \cdot\left(\tilde{\mathbf{S}}_{i} \times \tilde{\mathbf{S}}_{j}\right)\right], \\
& H_{D_{p}}=\sum_{\langle i j\rangle}-s_{i j} D_{p}\left[\sin (2 \eta) \sin \left(\frac{\theta_{i j}}{2}\right)\left(\tilde{S}_{i}^{z} \tilde{S}_{j}^{z}-\tilde{S}_{i}^{y} \tilde{S}_{j}^{y}\right)+\cos \eta \cos \left(\frac{\theta_{i j}}{2}\right) \hat{z} \cdot\left(\tilde{\mathbf{S}}_{i} \times \tilde{\mathbf{S}}_{j}\right)\right], \\
& H_{D_{R}}=\sum_{\langle i j\rangle}-s_{i j} v_{i j} D_{R}\left[\sin (2 \eta) \sin \left(\frac{\theta_{i j}}{2}\right)\left(\tilde{S}_{i}^{z} \tilde{S}_{j}^{z}-\tilde{S}_{i}^{y} \tilde{S}_{j}^{y}\right)+\cos \eta \cos \left(\frac{\theta_{i j}}{2}\right) \hat{z} \cdot\left(\tilde{\mathbf{S}}_{i} \times \tilde{\mathbf{S}}_{j}\right)\right] .
\end{aligned}
$$

Here we used the notation that $\theta_{i j}=\theta_{i}-\theta_{j}=-s_{i j} \frac{2 \pi}{3}, D_{z, i j}=D_{z} s_{i j}$, and $\mathbf{D}_{p, i j}=-s_{i j} D_{p}\left[\cos \left(\frac{\theta_{i}+\theta_{j}}{2}\right) \hat{x}+\sin \left(\frac{\theta_{i}+\theta_{j}}{2}\right) \hat{y}\right]$, where $s_{i j}$ is used to express the sign convention: $s_{i j}=1$ as the indices $i, j$ run clockwise around the triangle loop and $s_{i j}=-1$ when they run counterclockwise. The notation $v_{i j}$ takes care of the opposite convention for Rashba-DMI in upward and downward triangles with $v_{i j}= \pm 1$ for $(i j) \in \triangle / \nabla$. Plugging in the expression of $\theta_{i j}$ and performing the Holstein-Primakoff transformation $\tilde{S}_{i}^{x}=\sqrt{\frac{S}{2}}\left(b_{i}^{\dagger}+b_{i}\right), \tilde{S}_{i}^{y}=i \sqrt{\frac{S}{2}}\left(b_{i}^{\dagger}-b_{i}\right), \tilde{S}_{i}^{z}=\left(S-b_{i}^{\dagger} b_{i}\right)$, we can obtain the nearest-neighbor interaction

$$
H_{\mathrm{NN}}=\frac{1}{2} S \sum_{\langle i j\rangle}\left[\left(\Delta_{1}^{(0)}+v_{i j} \Delta_{R}^{(0)}\right)\left(b_{i}^{\dagger} b_{i}+b_{j}^{\dagger} b_{j}\right)+\left(\Delta_{1, i j}+v_{i j} \Delta_{R, i j}\right) b_{i}^{\dagger} b_{j}+\text { H.c. }+\left(\Delta_{1}^{\prime}+v_{i j} \Delta_{R}^{\prime}\right) b_{i}^{\dagger} b_{j}^{\dagger}+\text { h.c. }\right]
$$

with

$$
\begin{aligned}
& \Delta_{1}^{(0)}=J_{1}\left(1-3 \sin ^{2} \eta\right)-\sqrt{3}\left[D_{z} \cos ^{2} \eta+D_{p} \sin (2 \eta)\right], \quad \Delta_{1, i j}=\Delta_{1}^{\mathrm{re}}+i s_{i j} \Delta_{1}^{\mathrm{im}}, \\
& \Delta_{1}^{\mathrm{re}}=\frac{1}{2}\left[\left(1-3 \sin ^{2} \eta\right) J_{1}+\sqrt{3}\left(1+\sin ^{2} \eta\right) D_{z}-\sqrt{3} \sin (2 \eta) D_{p}\right], \quad \Delta_{1}^{\mathrm{im}}=\cos \eta D_{p}+\sin \eta\left(D_{z}+\sqrt{3} J_{1}\right), \\
& \Delta_{1}^{\prime}=\frac{1}{2}\left[\cos ^{2} \eta\left(\sqrt{3} D_{z}-3 J_{1}\right)+\sqrt{3} \sin (2 \eta) D_{p}\right],
\end{aligned}
$$

and

$$
\Delta_{R}^{(0)}=-\sqrt{3} D_{R} \sin 2 \eta, \quad \Delta_{R, i j}=-\frac{\sqrt{3}}{2} D_{R} \sin (2 \eta)+i s_{i j} D_{R} \cos \eta, \quad \Delta_{R}^{\prime}=\frac{\sqrt{3}}{2} \sin (2 \eta) D_{R} .
$$

In a similar way, we get second-nearest neighbor interaction, i.e., the second-nearest exchange, as

$$
H_{\mathrm{NNN}}=\frac{1}{2} S \sum_{\langle\langle i j\rangle\rangle}\left[\Delta_{2}^{(0)}\left(b_{i}^{\dagger} b_{i}+b_{j}^{\dagger} b_{j}\right)+\Delta_{2, i j} b_{i}^{\dagger} b_{j}+\text { H.c. }+\Delta_{2}^{\prime} b_{i}^{\dagger} b_{j}^{\dagger}+\text { H.c. }\right]
$$

with

$$
\begin{aligned}
& \Delta_{2}^{(0)}=J_{2}\left(1-3 \sin ^{2} \eta\right), \quad \Delta_{2, i j}=\Delta_{2}^{\mathrm{re}}+i s_{i j} \Delta_{2}^{\mathrm{im}}, \quad \Delta_{2}^{\mathrm{re}}=\frac{1}{2}\left(1-3 \sin ^{2} \eta\right) J_{2}, \\
& \Delta_{2}^{\mathrm{im}}=\sqrt{3} \sin \eta J_{2}, \quad \Delta_{2}^{\prime}=-\frac{3}{2} \cos ^{2} \eta J_{2} .
\end{aligned}
$$


Let's denote $H_{\mathrm{NN}}$ and $H_{\mathrm{NNN}}$ by $H_{1}$ and $H_{2}$, respectively. The total Hamiltonian can be written as $H=H_{1}+H_{2}+H_{R}$. By performing Fourier transformation, $H_{m}(m=1,2)$ becomes

$$
\begin{aligned}
H_{m}= & \frac{S}{2} \sum_{\mathbf{r}, \alpha \beta} \sum_{\lambda= \pm 1} \frac{1}{2}\left\{\Delta_{m}^{(0)}\left[b_{\alpha}^{\dagger}(\mathbf{r}) b_{\alpha}(\mathbf{r})+b_{\beta}^{\dagger}\left(\mathbf{r}+\lambda \delta_{\alpha \beta}^{(m)}\right) b_{\beta}\left(\mathbf{r}+\lambda \delta_{\alpha \beta}^{(m)}\right)\right]+\Delta_{m, \alpha \beta} b_{\alpha}^{\dagger}(\mathbf{r}) b_{\beta}\left(\mathbf{r}+\lambda \boldsymbol{\delta}_{\alpha \beta}^{(m)}\right)+\right.\text { H.c. } \\
& \left.+\Delta_{m}^{\prime} b_{\alpha}^{\dagger}(\mathbf{r}) b_{\beta}^{\dagger}\left(\mathbf{r}+\lambda \delta_{\alpha \beta}^{(m)}\right)+\text { H.c. }\right\} \\
= & \frac{S}{2} \sum_{\mathbf{k}, \alpha \beta}\left[4 \Delta_{m}^{(0)} \delta_{\alpha \beta}+2 \Delta_{m, \alpha \beta} \cos \left(\mathbf{k} \cdot \delta_{\alpha \beta}^{(m)}\right)\right] b_{\alpha, \mathbf{k}}^{\dagger} b_{\beta, \mathbf{k}}+\Delta_{m}^{\prime} \cos \left(\mathbf{k} \cdot \delta_{\alpha \beta}^{(m)}\right)\left(b_{\alpha, \mathbf{k}}^{\dagger} b_{\beta,-\mathbf{k}}^{\dagger}+b_{\alpha, \mathbf{k}} b_{\beta,-\mathbf{k}}\right) .
\end{aligned}
$$

Here $\boldsymbol{\delta}_{12}^{(1)}=\mathbf{e}_{3}, \boldsymbol{\delta}_{23}^{(1)}=\mathbf{e}_{1}, \boldsymbol{\delta}_{31}^{(1)}=\mathbf{e}_{2}$ and $\boldsymbol{\delta}_{12}^{(2)}=\mathbf{e}_{3}^{\prime}, \boldsymbol{\delta}_{23}^{(2)}=\mathbf{e}_{1}^{\prime}, \boldsymbol{\delta}_{31}^{(2)}=\mathbf{e}_{2}^{\prime}$. We choose $\boldsymbol{\delta}_{\alpha \beta}^{(m)}=-\boldsymbol{\delta}_{\beta \alpha}^{(m)}$ and $\mathbf{e}_{1}=\left(-\frac{1}{2},-\frac{\sqrt{3}}{2}\right), \mathbf{e}_{2}=(1,0)$, $\mathbf{e}_{3}=\left(-\frac{1}{2}, \frac{\sqrt{3}}{2}\right), \mathbf{e}_{1}^{\prime}=\mathbf{e}_{2}-\mathbf{e}_{3}, \mathbf{e}_{2}^{\prime}=\mathbf{e}_{3}-\mathbf{e}_{1}, \mathbf{e}_{3}^{\prime}=\mathbf{e}_{1}-\mathbf{e}_{2}$. In a similar way, we can show

$$
H_{R}=\frac{S}{2} \sum_{\mathbf{k}, \alpha \beta} i 2 \Delta_{R, \alpha \beta} \sin \left(\mathbf{k} \cdot \delta_{\alpha \beta}^{(1)}\right) b_{\alpha, \mathbf{k}}^{\dagger} b_{\beta, \mathbf{k}}+i \Delta_{m}^{\prime} \sin \left(\mathbf{k} \cdot \delta_{\alpha \beta}^{(1)}\right)\left(b_{\alpha, \mathbf{k}}^{\dagger} b_{\beta,-\mathbf{k}}^{\dagger}+b_{\alpha, \mathbf{k}} b_{\beta,-\mathbf{k}}\right) \text {. }
$$

Finally, the Hamiltonian is expressed in the basis $\Psi_{\mathbf{k}}=\left(b_{1, \mathbf{k}}, b_{2, \mathbf{k}}, b_{3, \mathbf{k}}, b_{1,-\mathbf{k}}^{\dagger}, b_{2,-\mathbf{k}}^{\dagger}, b_{3,-\mathbf{k}}^{\dagger}\right)^{T}$ as $H=\frac{S}{2} \sum_{\mathbf{k}} \Psi_{\mathbf{k}} \mathcal{H}_{\mathbf{k}} \Psi_{\mathbf{k}}$ with

$$
\mathcal{H}_{\mathbf{k}}=\left(\begin{array}{cc}
A_{0}+A_{\mathbf{k}} & B_{\mathbf{k}} \\
B_{\mathbf{k}} & A_{0}+A_{\mathbf{k}}^{*}
\end{array}\right) \text {. }
$$

Here, $A_{0}=2\left(\Delta_{1}^{(0)}+\Delta_{2}^{(0)}\right) \mathbb{1}_{3 \times 3}$ and

$$
\begin{aligned}
A_{\mathbf{k}} & =\left(\begin{array}{ccc}
0 & \cos k_{3} \Delta_{1} & \cos k_{2} \Delta_{1}^{*} \\
\cos k_{3} \Delta_{1}^{*} & 0 & \cos k_{1} \Delta_{1} \\
\cos k_{2} \Delta_{1} & \cos k_{1} \Delta_{1}^{*} & 0
\end{array}\right)+\left(\begin{array}{ccc}
0 & \cos p_{3} \Delta_{2} & \cos p_{2} \Delta_{2}^{*} \\
\cos p_{3} \Delta_{2}^{*} & 0 & \cos p_{1} \Delta_{2} \\
\cos p_{2} \Delta_{2} & \cos p_{1} \Delta_{2}^{*} & 0
\end{array}\right)+\left(\begin{array}{ccc}
0 & i \sin k_{3} \Delta_{R} & -i \sin k_{2} \Delta_{R}^{*} \\
-i \sin k_{3} \Delta_{R}^{*} & 0 & i \sin k_{1} \Delta_{R} \\
i \sin k_{2} \Delta_{R} & -i \sin k_{1} \Delta_{R}^{*} & 0
\end{array}\right) \\
B_{\mathbf{k}} & =\Delta_{1}^{\prime}\left(\begin{array}{ccc}
0 & \cos k_{3} & \cos k_{2} \\
\cos k_{3} & 0 & \cos k_{1} \\
\cos k_{2} & \cos k_{1} & 0
\end{array}\right)+\Delta_{2}^{\prime}\left(\begin{array}{cccc}
0 & \cos p_{3} & \cos p_{2} \\
\cos p_{3} & 0 & \cos p_{1} \\
\cos p_{2} & \cos p_{1} & 0
\end{array}\right)+\Delta_{R}^{\prime}\left(\begin{array}{ccc}
0 & i \sin k_{3} & -i \sin k_{2} \\
-i \sin k_{3} & 0 & i \sin k_{1} \\
i \sin k_{2} & -i \sin k_{1} & 0
\end{array}\right) .
\end{aligned}
$$

We abbreviated the notations: $k_{i}=\mathbf{k} \cdot \mathbf{e}_{i}, p_{i}=\mathbf{k} \cdot \mathbf{e}_{i}^{\prime}, \Delta_{m}=$ $\Delta_{m}^{\mathrm{re}}+i \Delta_{m}^{\mathrm{im}} \quad(m=1,2), \quad \Delta_{R}=-\frac{\sqrt{3}}{2} D_{R} \sin (2 \eta)+i D_{R} \cos \eta$ and considered the convention that $s_{12}=s_{23}=s_{31}=1$ and $s_{i j}=-s_{j i}$.

\section{Breathing pyrochlore antiferromagnet}

We consider the model

$$
H=J \sum_{\langle i j\rangle \in u} \mathbf{S}_{\mathbf{r}_{i}} \cdot \mathbf{S}_{\mathbf{r}_{j}}+J^{\prime} \sum_{\langle i j\rangle \in d} \mathbf{S}_{\mathbf{r}_{i}} \cdot \mathbf{S}_{\mathbf{r}_{j}}+D \sum_{i}\left(\mathbf{S}_{\mathbf{r}_{i}} \cdot \hat{z}_{i}\right)^{2} .
$$

Similar to the two-dimensional model, the magnon excitation is represented via the local Holstein-Primakoff transformation as $\mathbf{S}_{\mu}=\left(S-a_{\mu}^{\dagger} a_{\mu}\right) \hat{z}_{\mu}+\sqrt{\frac{S}{2}}\left(a_{\mu}+a_{\mu}^{\dagger}\right) \hat{x}_{\mu}-i \sqrt{\frac{S}{2}}\left(a_{\mu}-\right.$ $\left.a_{\mu}^{\dagger}\right) \hat{y}_{\mu}$. Therefore, the exchange interaction between two neighboring spins is expressed as

$$
\begin{aligned}
\mathbf{S}_{\mu} \cdot \mathbf{S}_{\nu}= & S_{\mu}^{c} S_{\nu}^{d} \Lambda_{\mu \nu}^{c d}=S^{2} \Lambda_{\mu \nu}^{z z}-S\left(a_{\mu}^{\dagger} a_{\mu}+a_{\nu}^{\dagger} a_{v}\right) \Lambda_{\mu \nu}^{z z} \\
& +\frac{S}{2}\left[a_{\mu}^{\dagger} a_{\nu} \Gamma_{\mu \nu}+a_{\mu} a_{\nu} \Omega_{\mu \nu}+\text { H.c. }\right],
\end{aligned}
$$

where $\Gamma_{\mu \nu}=\Lambda_{\mu \nu}^{x x}+\Lambda_{\mu \nu}^{y y}-i \Lambda_{\mu \nu}^{x y}+i \Lambda_{\mu \nu}^{y x}$ and $\Omega_{\mu \nu}=\Lambda_{\mu \nu}^{x x}-$ $\Lambda_{\mu \nu}^{y y}-i \Lambda_{\mu \nu}^{x y}-i \Lambda_{\mu \nu}^{y x}$. Here $\Lambda_{\mu \nu}^{c d}=\hat{c}_{\mu} \cdot \hat{d}_{\nu}$ with $\hat{c}_{\mu}, \hat{d}_{\nu}$ being the $c, d$ axis of the local frame of $\mu$ and $\nu$ atoms, respectively, i.e., $c, d=x, y, z$ and $\mu, v \in(0,1,2,3)$ with $\mu \neq v$. We choose local frames as shown in Table II. It can be shown by straightforward calculation that $\Lambda_{\mu \nu}^{z z}=-\frac{1}{3}, \Gamma_{\mu \nu}=-\frac{2}{3}$, and $\Omega_{\mu \nu}=\frac{4}{3} e^{i \phi_{\mu \nu}}$ where $\phi_{01}=\phi_{23}=-\frac{\pi}{3}, \phi_{02}=\phi_{13}=\frac{\pi}{3}$, $\phi_{03}=\phi_{12}=\pi$, and other terms can be generated by $\phi_{\mu \nu}=$ $\phi_{\nu \mu}(\mu \neq v)$. By substituting the magnon representation of spin-spin interaction Eq. (B18) into Eq. (B17) and performing Fourier transformation, we obtain the noninteracting magnon Hamiltonian

$$
\begin{aligned}
H= & \sum_{\mathbf{k}, \mu v} S\left[\left(J+J^{\prime}-2 D\right) \delta_{\mu v}-\frac{1}{3}\left(J+J^{\prime} e^{-i \mathbf{k} \cdot \mathbf{d}_{\mu v}}\right)\right] a_{\mu, \mathbf{k}}^{\dagger} a_{v, \mathbf{k}} \\
& +S \frac{1}{3}\left(J+J^{\prime} e^{-i \mathbf{k} \cdot \mathbf{d}_{\mu v}}\right) e^{i \phi_{\mu v}} a_{\mu,-\mathbf{k}} a_{v, \mathbf{k}}+\text { H.c. },
\end{aligned}
$$

where $\mathbf{d}_{\mu \nu}=\mathbf{a}_{v}-\mathbf{a}_{\mu}$ with $\mathbf{a}_{0}=(0,0,0), \mathbf{a}_{1}=\frac{1}{2}(0,1,1)$ $\mathbf{a}_{2}=\frac{1}{2}(1,0,1)$, and $\mathbf{a}_{3}=\frac{1}{2}(1,1,0)$.

TABLE II. Local coordinates of AIAO breathing pyrochlore.

\begin{tabular}{cccc}
\hline \hline$\mu$ & $\hat{x}_{\mu}$ & $\hat{y}_{\mu}$ & $\hat{z}_{\mu}$ \\
\hline 0 & $\frac{1}{\sqrt{2}}(-1,1,0)$ & $\frac{1}{\sqrt{6}}(-1,-1,2)$ & $\frac{1}{\sqrt{3}}(1,1,1)$ \\
1 & $\frac{1}{\sqrt{2}}(-1,-1,0)$ & $\frac{1}{\sqrt{6}}(-1,1,-2)$ & $\frac{1}{\sqrt{3}}(1,-1,-1)$ \\
2 & $\frac{1}{\sqrt{2}}(1,1,0)$ & $\frac{1}{\sqrt{6}}(1,-1,-2)$ & $\frac{1}{\sqrt{3}}(-1,1,-1)$ \\
3 & $\frac{1}{\sqrt{2}}(1,-1,0)$ & $\frac{1}{\sqrt{6}}(1,1,2)$ & $\frac{1}{\sqrt{3}}(-1,-1,1)$ \\
\hline \hline
\end{tabular}


[1] I. Žutić, J. Fabian, and S. Das Sarma, Rev. Mod. Phys. 76, 323 (2004).

[2] A. Manchon, J. Železný, I. M. Miron, T. Jungwirth, J. Sinova, A. Thiaville, K. Garello, and P. Gambardella, Rev. Mod. Phys. 91, 035004 (2019).

[3] A. G. Aronov and Yu. B. Lyanda-Geller, JETP Lett. 50, 431 (1989).

[4] V. Edelstein, Solid State Commun. 73, 233 (1990).

[5] S. D. Ganichev, M. Trushin, and J. Schliemann, in Handbook of Spin Transport and Magnetism, edited by E. Y. Tsymbal and I. Žutić (CRC, Boca Raton, FL, 2011), p. 487.

[6] M. Trushin and J. Schliemann, Phys. Rev. B 75, 155323 (2007).

[7] A. Y. Silov, P. A. Blajnov, J. H. Wolter, R. Hey, K. H. Ploog, and N. S. Averkiev, Appl. Phys. Lett. 85, 5929 (2004).

[8] C. L. Yang, H. T. He, L. Ding, L. J. Cui, Y. P. Zeng, J. N. Wang, and W. K. Ge, Phys. Rev. Lett. 96, 186605 (2006).

[9] N. P. Stern, S. Ghosh, G. Xiang, M. Zhu, N. Samarth, and D. D. Awschalom, Phys. Rev. Lett. 97, 126603 (2006).

[10] P. Gambardella and I. M. Miron, Philos. Trans. R. Soc. London A 369, 3175 (2011).

[11] J.-i. Inoue, G. E. W. Bauer, and L. W. Molenkamp, Phys. Rev. B 67, 033104 (2003).

[12] K. Shen, G. Vignale, and R. Raimondi, Phys. Rev. Lett. 112, 096601 (2014).

[13] J. Borge, C. Gorini, G. Vignale, and R. Raimondi, Phys. Rev. B 89, 245443 (2014).

[14] A. Johansson, J. Henk, and I. Mertig, Phys. Rev. B 93, 195440 (2016).

[15] C. Gorini, A. Maleki Sheikhabadi, K. Shen, I. V. Tokatly, G. Vignale, and R. Raimondi, Phys. Rev. B 95, 205424 (2017).

[16] S. D. Ganichev, E. L. Ivchenko, V. V. Bel'kov, S. A. Tarasenko, M. Sollinger, D. Weiss, W. Wegscheider, and W. Prettl, Nature (London) 417, 153 (2002).

[17] I. Garate and M. Franz, Phys. Rev. Lett. 104, 146802 (2010).

[18] A. Chernyshov, M. Overby, X. Liu, J. K. Furdyna, Y. LyandaGeller, and L. P. Rokhinson, Nat. Phys. 5, 656 (2009).

[19] I. M. Miron, G. Gaudin, S. Auffret, B. Rodmacq, A. Schuhl, S. Pizzini, J. Vogel, and P. Gambardella, Nat. Mater. 9, 230 (2010).

[20] I. M. Miron, K. Garello, G. Gaudin, P.-J. Zermatten, M. V. Costache, S. Auffret, S. Bandiera, B. Rodmacq, A. Schuhl, and P. Gambardella, Nature (London) 476, 189 (2011).

[21] D. A. Pesin and A. H. MacDonald, Phys. Rev. B 86, 014416 (2012)

[22] A. Qaiumzadeh, R. Â. A. Duine, and M. Titov, Phys. Rev. B 92, $014402(2015)$.

[23] I. A. Ado, O. A. Tretiakov, and M. Titov, Phys. Rev. B 95, 094401 (2017).

[24] K. D. Belashchenko, A. A. Kovalev, and M. van Schilfgaarde, Phys. Rev. Mater. 3, 011401 (2019).

[25] I. Dzyaloshinsky, J. Phys. Chem. Solids 4, 241 (1958).

[26] T. Moriya, Phys. Rev. 120, 91 (1960).

[27] N. Okuma, Phys. Rev. Lett. 119, 107205 (2017).

[28] A. Manchon, P. B. Ndiaye, J.-H. Moon, H.-W. Lee, and K.-J. Lee, Phys. Rev. B 90, 224403 (2014).

[29] A. A. Kovalev and V. Zyuzin, Phys. Rev. B 93, 161106(R) (2016).

[30] A. A. Kovalev, V. A. Zyuzin, and B. Li, Phys. Rev. B 95, 165106 (2017).

[31] H. Katsura, N. Nagaosa, and P. A. Lee, Phys. Rev. Lett. 104, 066403 (2010).
[32] Y. Onose, T. Ideue, H. Katsura, Y. Shiomi, N. Nagaosa, and Y. Tokura, Science 329, 297 (2010).

[33] R. Matsumoto and S. Murakami, Phys. Rev. Lett. 106, 197202 (2011).

[34] R. Matsumoto and S. Murakami, Phys. Rev. B 84, 184406 (2011).

[35] R. Matsumoto, R. Shindou, and S. Murakami, Phys. Rev. B 89, 054420 (2014).

[36] P. Laurell and G. A. Fiete, Phys. Rev. B 98, 094419 (2018).

[37] Y. Lu, X. Guo, V. Koval, and C. Jia, Phys. Rev. B 99, 054409 (2019).

[38] S. A. Owerre, Phys. Rev. B 95, 014422 (2017).

[39] H. Doki, M. Akazawa, H.-Y. Lee, J. H. Han, K. Sugii, M. Shimozawa, N. Kawashima, M. Oda, H. Yoshida, and M. Yamashita, Phys. Rev. Lett. 121, 097203 (2018).

[40] A. Mook, J. Henk, and I. Mertig, Phys. Rev. B 99, 014427 (2019).

[41] A. Mook, J. Henk, and I. Mertig, Phys. Rev. B 94, 174444 (2016).

[42] S. K. Kim, K. Nakata, D. Loss, and Y. Tserkovnyak, Phys. Rev. Lett. 122, 057204 (2019).

[43] V. A. Zyuzin and A. A. Kovalev, Phys. Rev. Lett. 117, 217203 (2016).

[44] R. Cheng, S. Okamoto, and D. Xiao, Phys. Rev. Lett. 117, 217202 (2016).

[45] Y. Shiomi, R. Takashima, and E. Saitoh, Phys. Rev. B 96, 134425 (2017).

[46] B. Li, S. Sandhoefner, and A. A. Kovalev, Phys. Rev. Research 2, 013079 (2020).

[47] A. Mook, R. R. Neumann, J. Henk, and I. Mertig, Phys. Rev. B 100, 100401(R) (2019).

[48] Y. Zhang, S. Okamoto, and D. Xiao, Phys. Rev. B 98, 035424 (2018).

[49] V. A. Zyuzin and A. A. Kovalev, Phys. Rev. B 97, 174407 (2018).

[50] A. Mook, B. Göbel, J. Henk, and I. Mertig, Phys. Rev. B 97, 140401(R) (2018).

[51] K. Nakata, S. K. Kim, J. Klinovaja, and D. Loss, Phys. Rev. B 96, 224414 (2017).

[52] M. W. Daniels, W. Yu, R. Cheng, J. Xiao, and D. Xiao, Phys. Rev. B 99, 224433 (2019).

[53] B. Li, A. Mook, and A. Kovalev, in APS Meeting Abstracts, Los Angeles, California (2018), p. R22.014, http://meetings. aps.org/Meeting/MAR18/Session/R22.14.

[54] J. M. Luttinger, Phys. Rev. 135, A1505 (1964).

[55] T. Holstein and H. Primakoff, Phys. Rev. 58, 1098 (1940).

[56] C. Psaroudaki, S. Hoffman, J. Klinovaja, and D. Loss, Phys. Rev. X 7, 041045 (2017).

[57] To simplify notations, the magnonic spin-density matrix is defined here with additional factor $1 / 2$ compared to Ref. [46]. However, the overall definition of the spin-density operator is equivalent to Ref. [46].

[58] A. Shitade, A. Daido, and Y. Yanase, Phys. Rev. B 99, 024404 (2019).

[59] J. Shi, G. Vignale, D. Xiao, and Q. Niu, Phys. Rev. Lett. 99, 197202 (2007).

[60] L. Dong, C. Xiao, B. Xiong, and Q. Niu, arXiv:1812.11721 (2018).

[61] M. E. Zhitomirsky and A. L. Chernyshev, Rev. Mod. Phys. 85, 219 (2013) 
[62] A. L. Chernyshev and M. E. Zhitomirsky, Phys. Rev. B 92, 144415 (2015).

[63] S. Streib, N. Vidal-Silva, K. Shen, and G. E. W. Bauer, Phys. Rev. B 99, 184442 (2019).

[64] T. Kikkawa, K. Shen, B. Flebus, R. A. Duine, K.-i. Uchida, Z. Qiu, G. E. W. Bauer, and E. Saitoh, Phys. Rev. Lett. 117, 207203 (2016).

[65] S. Park and B.-J. Yang, Phys. Rev. B 99, 174435 (2019).

[66] M. Seemann, D. Ködderitzsch, S. Wimmer, and H. Ebert, Phys. Rev. B 92, 155138 (2015).

[67] J. Železný, H. Gao, A. Manchon, F. Freimuth, Y. Mokrousov, J. Zemen, J. Mašek, J. Sinova, and T. Jungwirth, Phys. Rev. B 95, 014403 (2017).

[68] M. Kimata, H. Chen, K. Kondou, S. Sugimoto, P. K. Muduli, M. Ikhlas, Y. Omori, T. Tomita, A. H. MacDonald, S. Nakatsuji et al., Nature (London) 565, 627 (2019).

[69] J. Železný, H. Gao, K. Výborný, J. Zemen, J. Mašek, A. Manchon, J. Wunderlich, J. Sinova, and T. Jungwirth, Phys. Rev. Lett. 113, 157201 (2014).

[70] L. Salemi, M. Berritta, A. K. Nandy, and P. M. Oppeneer, Nat. Commun. 10, 5381 (2019).

[71] A. Kamra, U. Agrawal, and W. Belzig, Phys. Rev. B 96, 020411(R) (2017).

[72] U. Ritzmann, Ph.D. thesis, University of Konstanz, Konstanz, 2015.

[73] F. Hellman, A. Hoffmann, Y. Tserkovnyak, G. S. D. Beach, E. E. Fullerton, C. Leighton, A. H. MacDonald, D. C. Ralph, D. A. Arena, H. A. Dürr et al., Rev. Mod. Phys. 89, 025006 (2017).

[74] A. L. Chernyshev and M. E. Zhitomirsky, Phys. Rev. Lett. 113, 237202 (2014).

[75] R. Okuma, T. Yajima, D. Nishio-Hamane, T. Okubo, and Z. Hiroi, Phys. Rev. B 95, 094427 (2017).

[76] S. Toth and B. Lake, J. Phys.: Condens. Matter 27, 166002 (2015).

[77] K. Matan, D. Grohol, D. G. Nocera, T. Yildirim, A. B. Harris, S. H. Lee, S. E. Nagler, and Y. S. Lee, Phys. Rev. Lett. 96, 247201 (2006).
[78] S. K. Kim and Y. Tserkovnyak, Phys. Rev. B 92, 020410(R) (2015).

[79] F.-Y. Li, Y.-D. Li, Y. B. Kim, L. Balents, Y. Yu, and G. Chen, Nat. Commun. 7, 12691 (2016).

[80] F.-Y. Li and G. Chen, Phys. Rev. B 98, 045109 (2018).

[81] S.-K. Jian and W. Nie, Phys. Rev. B 97, 115162 (2018).

[82] K. Hwang, N. Trivedi, and M. Randeria, arXiv:1712.08170.

[83] Y. Okamoto, G. J. Nilsen, J. P. Attfield, and Z. Hiroi, Phys. Rev. Lett. 110, 097203 (2013).

[84] F. Casola, T. van der Sar, and A. Yacoby, Nat. Rev. Mater. 3, 17088 (2018).

[85] W. F. Brown, Phys. Rev. 130, 1677 (1963).

[86] R. F. L. Evans, W. J. Fan, P. Chureemart, T. A. Ostler, M. O. A. Ellis, and R. W. Chantrell, J. Phys.: Condens. Matter 26, 103202 (2014).

[87] J. Barker and G. E. W. Bauer, Phys. Rev. Lett. 117, 217201 (2016).

[88] D. Inosov, Adv. Phys. 67, 149 (2018).

[89] A. L. Chernyshev and M. E. Zhitomirsky, Phys. Rev. B 79, 144416 (2009).

[90] M. V. Gvozdikova, P.-E. Melchy, and M. E. Zhitomirsky, J. Phys.: Condens. Matter 23, 164209 (2011).

[91] L. E. Svistov, A. I. Smirnov, L. A. Prozorova, O. A. Petrenko, L. N. Demianets, and A. Y. Shapiro, Phys. Rev. B 67, 094434 (2003).

[92] J. Hwang, E. S. Choi, F. Ye, C. R. Dela Cruz, Y. Xin, H. D. Zhou, and P. Schlottmann, Phys. Rev. Lett. 109, 257205 (2012).

[93] J. G. Rau, L. S. Wu, A. F. May, L. Poudel, B. Winn, V. O. Garlea, A. Huq, P. Whitfield, A. E. Taylor, M. D. Lumsden et al., Phys. Rev. Lett. 116, 257204 (2016).

[94] T. Haku, K. Kimura, Y. Matsumoto, M. Soda, M. Sera, D. Yu, R. A. Mole, T. Takeuchi, S. Nakatsuji, Y. Kono, T. Sakakibara, L.-J. Chang, and T. Masuda, Phys. Rev. B 93, 220407(R) (2016).

[95] Y. Tanaka, M. Yoshida, M. Takigawa, Y. Okamoto, and Z. Hiroi, Phys. Rev. Lett. 113, 227204 (2014).

[96] A. Shitade and Y. Yanase, Phys. Rev. B 100, 224416 (2019). 